Journal of Fluid Mechanics

http://journals.cambridge.org/FLM

Journal of

Additional services for Journal of Fluid Mechanics:

Fluid Mechanics

Email alerts: $\underline{\text { Click here }}$

Subscriptions: $\underline{\text { Click here }}$

Commercial reprints: $\underline{\text { Click here }}$

Terms of use : $\underline{\text { Click here }}$

\title{
Unsteady separation past moving surfaces
}

A. T. DEGANI, J. D. A. WALKER and F. T. SMITH

Journal of Fluid Mechanics / Volume 375 / November 1998, pp 1 - 38

DOI: 10.1017/S0022112098001839, Published online: 08 September 2000

Link to this article: http://journals.cambridge.org/abstract_S0022112098001839

How to cite this article:

A. T. DEGANI, J. D. A. WALKER and F. T. SMITH (1998). Unsteady separation past moving surfaces. Journal of Fluid Mechanics, 375, pp 1-38 doi:10.1017/S0022112098001839

Request Permissions : $\underline{\text { Click here }}$ 
(C) 1998 Cambridge University Press

\title{
Unsteady separation past moving surfaces
}

\author{
By A. T. DEGANI $I^{1}$, J. D. A. WALKER ${ }^{1}$ AND F. T. SMITH ${ }^{2}$ \\ ${ }^{1}$ Department of Mechanical Engineering and Mechanics, 354 Packard Laboratory, \\ 19 Memorial Drive West, Lehigh University, Bethlehem, PA 18015, USA \\ 2 Department of Mathematics, University College London, Gower Street, \\ London WCIE 6BT, UK
}

(Received 9 October 1993 and in revised form 3 April 1998)

\begin{abstract}
Unsteady boundary-layer development over moving walls in the limit of infinite Reynolds number is investigated using both the Eulerian and Lagrangian formulations. To illustrate general trends, two model problems are considered, namely the translating and rotating circular cylinder and a vortex convected in a uniform flow above an infinite flat plate. To enhance computational speed and accuracy for the Lagrangian formulation, a remeshing algorithm is developed. The calculated results show that unsteady separation is delayed with increasing wall speed and is eventually suppressed when the speed of the separation singularity approaches that of the local mainstream velocity. This suppression is also described analytically. Only 'upstreamslipping' separation is found to occur in the model problems. The changes in the topological features of the flow just prior to the separation that occur with increasing wall speed are discussed.
\end{abstract}

\section{Introduction}

Unsteady boundary-layer flow separation at high Reynolds numbers occurs in an extensive number of flow configurations (Van Dommelen 1981; Cowley, Van Dommelen \& Lam 1990; Doligalski, Smith \& Walker 1994). Examples of such phenomena occur in (i) flow past surface-mounted obstacles, such as wing-body junctions, (ii) flow on the suction side of a thin airfoil aligned at an angle of attack to the mainstream, (iii) vortex interactions with surfaces, such as tip vortex impingement on the tail/fuselage of aircraft and wake-rotor interactions in turbines, (iv) impulsive motion of bluff bodies and (v) flow in internal passages with obstructions. At smaller scales, unsteady separation is also believed to be the mechanism whereby new turbulence is generated within a turbulent boundary layer (Smith et al. 1991; Smith \& Walker 1995). In some of the above examples, unsteady separation occurs in a complex three-dimensional environment and may involve associated phenomena such as turbulence and compressibility. However, the basic mechanism of unsteady separation at high Reynolds numbers is believed to be similar in most such flows (Van Dommelen \& Cowley 1990; Cowley et al. 1990). In many circumstances, a recirculation zone develops in the boundary layer within a region of local adverse streamwise pressure gradient, and this event heralds a subsequent chain of occurrences leading to unsteady separation of the boundary layer from the surface. Eventually, a narrow region with sharp streamwise gradients and strong upwelling develops upstream of the recirculation zone; this region rapidly thins in the streamwise direction with time and ultimately provokes a viscous-inviscid interaction, wherein a narrow plume containing significant levels 
of vorticity is ejected from within the boundary layer into the external mainstream inviscid flow (Cowley et al. 1990; Smith et al. 1991; Doligalski et al. 1994).

The problem of laminar boundary-layer separation at high Reynolds number $R e$ has traditionally been approached as being zonal in nature involving an interaction between an external region and a thin boundary layer adjacent to the body surface. In the classical interactive strategy (reviewed by Smith 1982, for example), the external inviscid flow is calculated first and the resulting pressure distribution near the body is prescribed in order to evaluate the boundary-layer flow. For steady flow past fixed walls, 'separation' was classically defined as being associated with the development of reversed flow and the location where the wall shear stress vanishes; however, a Goldstein singularity structure occurs in the solution of the steady boundarylayer equations at such points in situations where the pressure gradient is adverse and prescribed. For certain steady boundary-layer flows past fixed walls, a selfconsistent asymptotic structure can be constructed using interactive concepts (see, for example, Smith 1982); this physical description normally involves a multi-layer structure (often containing regions of reversed flow) and an external mainstream flow that is displaced an $o(1)$ distance from the wall (in the limit $R e \rightarrow \infty$ ) via a localized viscous-inviscid interaction. On the other hand, for unsteady flows and steady flow past moving walls, the situation is more complicated and Moore (1958), Rott (1957) and Sears (1956) concluded independently that vanishing wall shear and reversed flow were not a necessarily meaningful (and certainly not general) criteria for flow separation. Subsequently, Sears \& Telionis (1975) postulated the MRS model of unsteady separation, in which 'separation' was defined as that instant when a singularity evolves in the solution of the boundary-layer equations. In physical terms, this breakdown of the boundary-layer solution indicates the first instant when a hitherto thin and passive shear layer adjacent to the wall starts to interact with external flow and thereby separate from the surface. In motion which is impulsively started from rest, the boundary layer on a body is initially thin and passively driven by the pressure distribution impressed by the external potential flow; this situation generally does not persist, particularly when strong local outflows develop, and if the pressure gradient is prescribed for an indefinite period of time, the boundary-layer solution responds by developing a singularity. This irregular behaviour signals the onset of an interaction with the external flow and indicates the need to consider a more complex subset of the Navier-Stokes equations in order to describe locally the developing boundary-layer eruption.

Sears \& Telionis (1975) argued that separation would generally occur at a streamwise location $x_{s}$ somewhere in the middle of the boundary where two MRS conditions are satisfied, namely (i) MRS I - the streamwise velocity vanishes in a frame of reference moving with the separation (i.e. the local particle velocity $u$ ), and (ii) MRS II the vorticity $\omega=-\partial u / \partial y$ vanishes at $x=x_{s}$. As discussed by Sears \& Telionis (1975), the evolution of a singularity in the boundary-layer solution $(R e \rightarrow \infty)$ delineates a definite time and location where the boundary layer separates from the surface and consequently is an important diagnostic tool for unsteady flows at finite Re. According to the MRS model, separation must eventually occur somewhere along a line of zero vorticity; such lines are often associated with the prior development of recirculation as a consequence of viscous effects operating in regions of adverse pressure gradient within the boundary layer. A clear discussion of the separation process and the significance of the zero vorticity line $(\omega=0)$ has been given by Van Dommelen (1991), and some central points are repeated here. At times and locations close to separation, the boundary-layer flow develops strong local vertical currents and thickens rapidly; 

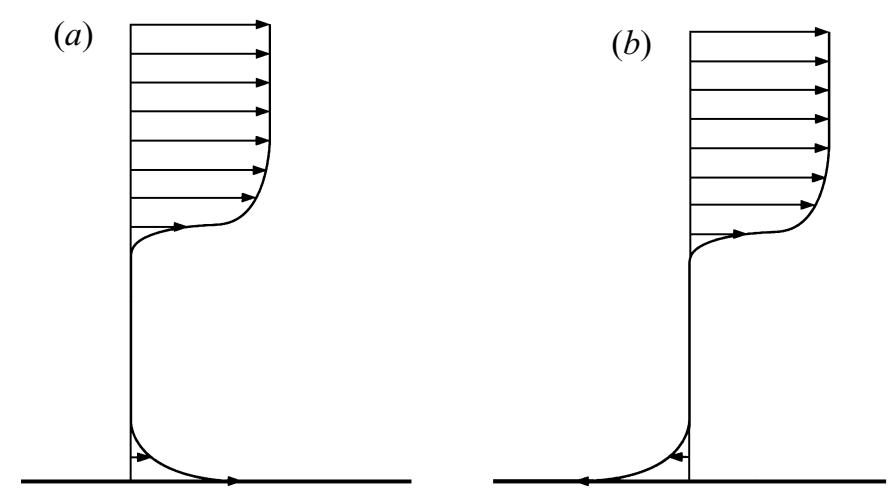

FIGURE 1. Streamwise velocity profile at separation: (a) upstream-slipping separation (downstream-moving wall) and $(b)$ downstream-slipping separation (upstream-moving wall).

the motion in the upper portion of the boundary layer is effectively inviscid and the streamwise momentum equation for the particle velocities $u$ becomes arbitrarily close to the unsteady one-dimensional Burgers' equation $\partial u / \partial t+u \partial u / \partial x=0$ along the zero vorticity line; in Lagrangian coordinates, this equation implies that the streamwise velocity of fluid particles is conserved on the line $\omega=0$. However, vorticity remains constant at a particle embedded in an inviscid flow and therefore the line $\omega=0$ is also a material line. It follows that, in a situation where the values of the particle velocities $u$ are different on the line $\omega=0$ at some instant prior to separation, a process of compression must occur in which adjacent fluid particles approach each other arbitrarily closely in a finite time. This process leads to the severe normal growth (away from the wall) of a particular fluid particle at the instant of separation (see also Cowley et al. 1990).

In any experiment or calculation, it is relatively easy to identify lines of zero vorticity, and since the location of $\omega=0$ is invariant to a Galilean transformation, the particular reference frame of the observer is not at issue (in contrast to the first MRS condition). As discussed by Williams (1977) and Van Dommelen (1981), MRS I is less useful in establishing the location of separation since the speed of the separation structure is not known a priori; moreover, it is essentially impossible to evaluate $u_{s}$ in a conventional Eulerian formulation. In any event, Sears \& Telionis (1975) identified two possible situations for which the streamwise velocity profiles are sketched in figure 1 . In both cases the external mainstream velocity is from left to right and the velocity profile at separation is sketched in a frame of reference moving with the separation (i.e. the local flow speed at a location where $\omega=0$ ). As shown in figure 1 there are two possibilities which were described as the 'downstreammoving' and 'upstream-moving' wall respectively; in this terminology, the phenomena are categorized according to the motion of the wall relative to that of the separation structure. Another similar terminology has also been introduced (Williams 1977; Van Dommelen 1981) which will be used throughout the present paper. Each case is described in terms of the relative motion of the separation singularity relative to the wall; in this nomenclature the situations in figures $1(a)$ and $1(b)$ are referred to as 'upstream-slipping' and 'downstream-slipping' separation, respectively. In principle, both cases are possible.

Sears \& Telionis (1975) also proposed that the unsteady separation singularity would be similar to the Goldstein singularity. A number of numerical studies were conducted (see, for example, Tsahalis 1977; Telionis, Tshalis \& Werle 1973; and 
Telionis \& Werle 1973) to validate the MRS-criterion of flow separation. However, the calculations encountered convergence difficulties when severe variations developed in the flow field and none of the computational schemes were able to advance the computations up to the point of occurrence of the MRS condition. Subsequently, a major advance was made by Van Dommelen (1981) and Van Dommelen \& Shen $(1980,1982)$, who recast the boundary-layer equations in a Lagrangian coordinate system; in this manner an unambiguous criterion for the location and occurrence of the unsteady separation singularity was eventually established.

Van Dommelen \& Shen $(1980,1982)$ carried out numerical solutions in the Lagrangian formulation for the boundary layer on an impulsively started circular cylinder; they established that a separation singularity occurs at $t_{s}=1.5$ (in dimensionless time) and at $x_{s}=1.94$, where $x$ is dimensionless arclength measured along the cylinder from the front stagnation point. Here the non-dimensionalizations are based on the radius and translational speed of the cylinder. It was found that the MRS conditions were satisfied at separation which was determined to be of the upstreamslipping type. The analytical structure of this type of separation was considered by Van Dommelen \& Shen (1982) and Elliott, Smith \& Cowley (1983) and found to be substantially different from the Goldstein singularity. In the unsteady structure, the boundary layer bifurcates into two shear layers on either side of separation, with one shear layer remaining adjacent to the wall and the other growing rapidly away from the surface; the shear layers bound an inviscid region where the vorticity is almost zero. The separation process is driven by the dynamics of the central inviscid region where fluid particles are compressed along the zero vorticity line and grow explosively in a direction normal to the surface as $t \rightarrow t_{s}$. The analysis of Elliott et al. (1983) suggests that the asymptotic structure for upstream-slipping separation is generic in two-dimensional flows, and a similar behaviour was subsequently found (using a Lagrangian formulation) in problems relating to separation on a rotating and translating cylinder at moderate rotation speeds (Shen \& Wu 1990) and in vortex-induced separation (Peridier, Smith \& Walker 1991).

To date, numerical solutions have not been produced for a physical problem in which it can be categorically stated that downstream-slipping separation occurs. It should be emphasized that in order to definitively resolve such issues, it is necessary to be able to complete a calculation through to $t_{s}$, and at present Lagrangian calculations are the only schemes which have been successful in this endeavour. Admittedly the solution set and the number of physical problems treated by Lagrangian methods is small as yet but, if cases of downstream-slipping do exist, it appears they may be significantly rarer than upstream-slipping separation. In the vortex-induced separation problem considered by Peridier et al. (1991), a profile at separation was obtained which at first glance appeared similar to that in figure $1(b)$. However, on close examination the separation profile was found to exhibit a local maximum near the wall, and the growth of the boundary layer was found to be $O\left(t_{s}-t\right)^{-1 / 4}$, matching that of the upstream-slipping singularity structure described by Van Dommelen \& Shen (1982) and Elliott et al. (1983). Nevertheless the downstream-slipping case has been of historical interest (Williams 1977), as well as being controversial, and local analyses have been carried out by Van Dommelen (1981) and Van Dommelen \& Shen (1983) in an effort to describe such a structure. In addition, sketches of the anticipated streamline patterns expected near the singular structure have been given by Sears \& Telionis (1975) and subsequently modified by Van Dommelen (1981). At present the case of upstream-slipping separation seems well verified, but as Williams (1977) remarks, the nature of downstream-slipping separation is still unknown. 
The problem of a cylinder rotating in a uniform stream has been of historical interest since the wall moves in the direction of the external flow as well as counter to it on various portions of the cylinder. Thus some authors expected that both types of separation might occur in this geometry. Koromilas \& Telionis (1980) carried out flow visualization studies for a rotating cylinder at low Reynolds number and observed flow patterns near the upper and lower surface similar to those sketched by Sears \& Telionis (1975). The case of downstream-slipping separation is believed to be associated with a saddle point in the instantaneous streamline patterns (Van Dommelen 1981) and such behaviour was also noted in the boundary-layer calculations of Ece, Walker \& Doligalski (1984) for high-speed flow past a rotating cylinder; however, the latter calculations were done using a spectral method in an Eulerian formulation and could not be extended through to the formation of a singularity. Thus the issue of whether downstream-slipping separation occurs in the rotating cylinder problem is not as yet resolved.

In the present study, Lagrangian calculations were carried out for the impulsively started translating and rotating cylinder for a range of rotation rates. For low to moderate rotation rates, a singularity develops, but it is always of the upstreamslipping type, and no cases of downstream-slipping separation were found. A second important issue of interest here relates to the manner in which separation is suppressed by a moving wall; here and throughout the paper, 'unsteady separation' refers to the occurrence of a finite-time singularity in the boundary-layer solution. A moving surface is known to act to inhibit the separation process (see, for example, Ece et al. 1984). However, the minimum wall speed required to achieve suppression is not known, and here the process through which this occurs will be addressed through consideration of two very different model problems. Interestingly, it will be shown that elimination of upstream-slipping separation occurs through a similar process in both cases as the wall speed is increased, suggesting a generic type of behaviour that generally does not involve any cases of downstream-slipping separation.

The plan of the paper is as follows. The model problems are described in $\S 2$, while the governing equations and numerical methods are discussed in $\S 3$ and $\S 4$ respectively (see also Appendices A and B). Calculated results for the rotating cylinder and vortex problems are given in $\S 5$ and $\S 6$, respectively, and an analytical account of how the unsteady separation singularity is suppressed, at either a finite rotation rate of the wall or finite vortex convection speed, is presented in Appendix C. Finally, conclusions are drawn in $\S 7$.

\section{Model problems}

Two main objectives of this study are to determine the minimum wall speeds necessary to completely inhibit boundary-layer separation in the model problems and to understand the changes in unsteady flow topology that occur with increasing wall speed. The model problems consist of the unsteady boundary layer developing on: (i) an impulsively started translating and rotating circular cylinder, and (ii) an infinite plane wall, induced by a vortex convected in a uniform flow above the surface. In the former problem the wall moves in the laboratory frame while in the latter case, the wall appears to move in a frame of reference affixed to the vortex. Both examples represent a family of problems involving a single parameter $\beta$ that is proportional to the dimensionless wall speed. Although the physical origins of these problems are significantly different, separation is eventually suppressed at a critical value of $\beta$ in a manner which emerges to be essentially the same. 


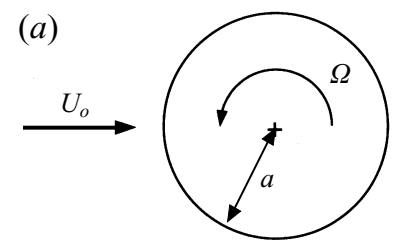

(b)

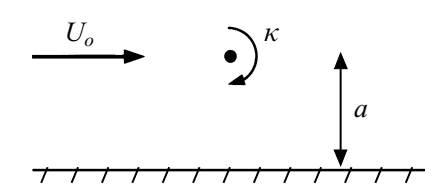

FiguRE 2. Geometry for the model problems: $(a)$ rotating and translating cylinder and $(b)$ convected vortex above a wall.

Consider first the cylinder problem shown schematically in figure 2(a). The cylinder has radius $a$ and rotates in the counter-clockwise direction while the translational speed is $U_{o}$. If all lengths and velocities are made dimensionless with respect to $a$ and $U_{o}$ respectively, the external inviscid flow distribution and dimensionless wall speed are given by

$$
U_{e}(x)=2 \sin x, \quad \beta=a \Omega / U_{o},
$$

respectively. Here $x$ measures dimensionless arclength in the clockwise direction from the front inviscid stagnation point. It is evident from equation (1) that an adverse pressure gradient is impressed on the boundary layer in the first and fourth quadrants; here quadrants of the plane are delineated with respect to a Cartesian coordinate system defined by the downstream radius $(x=\pi)$ and the vertical radius $x=\pi / 2$.

The rotating cylinder problem has been the subject of a number of theoretical and experimental studies. The case of very large rotation rates has been considered by Glauert (1957) and Moore (1957), who assumed that $\beta$ was large enough so that separation was completely suppressed; the situation addressed by these authors is relevant at large times after the initiation of the motion, since it was assumed that the external flow had acquired a steady circulation at some previous stage (presumably via a prior interaction with the developing boundary-layer flow on the cylinder). In any event, a steady boundary-layer solution was found describing an attached flow confined to a thin ring ensheathing the rapidly rotating cylinder. The solution is valid in the limit $\beta \rightarrow \infty$, but is expected to describe situations for large but finite values of $\beta$; since the case $\beta=0$ yields an inherently unsteady flow, it is expected that there is some critical large value of $\beta$ below which Glauert's (1957) solution does not apply. This type of question has been addressed in the work of Nikolayev (1982), Sychev (1987), Lam (1988) and Chipman \& Duck (1993) who studied, in effect, the minimum value of $\beta$ for which a steady periodic solution could be found. A careful numerical study by Lam (1988) establishes this minimum value as $\beta \simeq 3.906$ for an external flow containing circulation.

The rotating cylinder problem can be approached from another perspective by considering the impulsively started case and attempting to track the evolution forward in time, possibly through the viscous-inviscid interaction that ultimately produces circulation in the external flow for large $\beta$. Ece et al. (1984) calculated the initial development for the boundary layer on an impulsively started rotating and translating cylinder for various values of $\beta$ ranging from 0.05 to 10 using a Eulerian formulation. Although it is not possible to continue a Eulerian calculation all the way to the evolution of a separation singularity (Van Dommelen \& Shen 1980, 1982), Ece et al. (1984) were able to infer that unsteady separation is inhibited with increasing wall speed and noted the evolution of streamline patterns similar to those proposed by Sears \& Telionis (1975) near the MRS point for both upstream- and downstreammoving walls. Similar streamline patterns were also noted in the flow visualization 
studies of Koromilas \& Telionis (1980) at a free-stream Reynolds number of 50. The rotating cylinder problem has been of particular interest since, for the situation shown in figure 2(a), the external velocity $U_{e}$ and wall velocity $\beta$ are in opposite directions on the upper half of the cylinder and in the same direction on the lower half; thus it is conceivable that both upstream- and downstream-moving wall separation are possible for this problem. The streamline pattern conjectured by Sears \& Telionis (1975) for an upstream-moving wall was also observed in the computations of Ece et al. (1984) at $\beta=2$ and 10; however, at these values of $\beta$, the boundary layer was observed to thicken gradually without the presence of sharp upflows that were seen to start to develop for cases with $\beta<2$. It appears that for $\beta>2$, the thickening boundary layer could ultimately give rise to a smooth interaction with the outer flow in which the external region may possibly acquire circulation. Note that here our reference to a thickening boundary layer does not imply the existence of a steady limit.

For $\beta<2$, the boundary-layer response appears to be much more localized and abrupt. In a recent study, Shen $\& \mathrm{Wu}$ (1990) carried out calculations for $\beta=0.2$, 1.0 and 1.5 in Lagrangian coordinates and were able to continue up to the instant of unsteady separation. A sharp eruptive response was found to occur in the fourth quadrant in all cases (i.e. in the lower half-plane to the right; see figure $2 a$ ). It was determined that separation is delayed with increasing wall speed and is of the upstream-slipping type shown in figure $1(a)$. For the range of rotation rates considered, no downstream-slipping separation (shown in figure $1 b$ ) was observed. Since these calculations were part of a broader study on unsteady separation past bluff bodies, no attempt was made to determine the value of $\beta$ for which unsteady separation is suppressed or the manner in which this occurs.

The second model problem of interest here is shown schematically in figure $2(b)$ and was first considered by Doligalski \& Walker (1984). A vortex of negative strength $-\kappa$, which is located at a distance $a$ above an infinite plane wall, will be convected with uniform speed of $\kappa / 2 a$ (from right to left) through the influence of its image below the plate; if a uniform flow of speed $U_{o}$ is superimposed from left to right, the vortex convects to the right with a convection speed $V_{c}=U_{o}-\kappa / 2 a$. A fractional convection rate may be defined by $\alpha=V_{c} / U_{o}$, and it follows that $\alpha=1-\kappa /\left(2 a U_{o}\right)$ where the principal range of interest is $0 \leqslant \alpha<1$. At $\alpha=0$, the mainstream uniform flow exactly balances the self-induced velocity, and in this case the vortex remains stationary in a crossflow. The limit $\alpha \rightarrow 1$ corresponds to a situation where the self-induced speed of the vortex is vanishingly small in comparison with the uniform flow $U_{o}$. In a frame of reference moving with the vortex, the external flow field is steady, and the wall appears to move from right to left with speed $\alpha U_{o}$. It is convenient to non-dimensionalize velocities in terms of the mainstream velocity at infinity which is $(1-\alpha) U_{o}$ in a frame of reference convecting with the vortex. With this non-dimensionalization, the dimensionless external flow distribution and wall velocity may be shown to be given by (Doligalski \& Walker 1984)

$$
U_{e}=1-\frac{4}{1+x^{2}}, \quad \beta=\frac{\alpha}{1-\alpha},
$$

respectively, for all values of $\alpha$, in a frame of reference convecting uniformly with the vortex. Figure 3 shows a plot of $U_{e}$ and $\mathrm{d} U_{e} / \mathrm{d} x$. Stagnation points occur at $\pm \sqrt{3}$ and bracket a zone of flow in the negative $x$-direction; and for $|x|<\sqrt{3}$, the pressure gradient (with respect to the local velocity direction) is adverse for $x<0$ and 


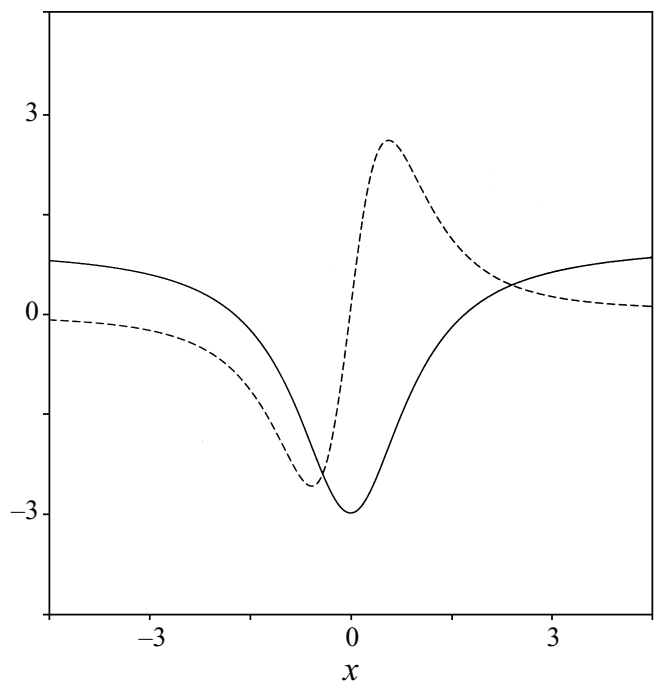

FIGURE 3. Mainstream velocity induced by the convected vortex in a frame of reference moving with the vortex:,$- U_{e} ;---, U_{e}^{\prime}$.

favourable for $x>0$. Between the rear stagnation point at $x=-\sqrt{3}$ and the vortex centre at $x=0$, the external velocity is in the same direction as the wall velocity.

The unsteady boundary-layer flow development following an impulsive start was considered by Doligalski \& Walker (1984) for a range of values from $\alpha=0$ to $\alpha=0.8$ (corresponding to $\beta=0$ and $\beta=4$, respectively). It was found that for values of $\alpha \leqslant 0.55$, a recirculating region of positive rotation developed in the boundary layer between $x=-\sqrt{3}$ and $x=0$. Subsequently, sharp upflows developed just upstream of the recirculation zone (in terms of the local mainstream velocity direction); however, the calculations could not be continued beyond this stage (in the Eulerian formulation) all the way through to what appeared to be a developing separation singularity. For increasing $\alpha$ (and hence $\beta$ ) the calculations could be carried on progressively further in time, and the strength of the eruptive motions seemed to diminish, suggesting that the tendency to unsteady separation is reduced with increasing $\beta$. In the range $0.55<\alpha \leqslant 0.75$, the formation of a recirculation zone did not occur, but upflows of relatively less intensity did develop which eventually terminated the calculations. Beyond $\alpha=0.75$, a gradual thickening of the boundary layer was observed over a streamwise extent $O(1)$, and it was concluded that unsteady separation is suppressed for $\alpha>0.75(\beta>3)$.

The cases of $\beta=2$ for the translating and rotating cylinder, and $\beta=3(\alpha=$ 0.75 ) for the convected vortex flow, correspond to apparently critical situations, in which the wall speed exactly balances the maximum magnitude of the external flow velocity (which is associated with the region of adverse pressure gradient). The calculations of Ece et al. (1984) and Doligalski \& Walker (1984) suggest that for values of $\beta$ beyond these critical values, the boundary layer gradually thickens with no evidence of sharp upflows. Thus in both cases when the wall speed is greater than the maximum magnitude of the mainstream velocity, the boundary-layer flow development appears to be dominated by the influence of the moving wall; this influence seems to mitigate the effects of the adverse mainstream pressure gradient, 
thereby leading to the suppression of unsteady separation. This conjectured behaviour will be discussed subsequently in more detail.

\section{Governing equations}

In the initial stages of the development of the unsteady boundary-layer flow, the solution is expected to be relatively smooth with an absence of sharp streamwise gradients; in general terms, an Eulerian formulation is most effective in such situations. For both model problems considered, an impulsive start is assumed which provides a well-defined initial condition in Eulerian coordinates; it is convenient to utilize this formulation from the impulsive start to some time $t=t_{o}$, where $t_{o}$ denotes a value well in advance of the expected instant of unsteady separation $t_{s}$. It is well-established that a boundary-layer calculation in Eulerian coordinates cannot be continued up to the instant of unsteady separation (Van Dommelen 1981; Peridier et al. 1991). On the other hand, the Lagrangian formulation is very effective in resolving the flow field when separation begins to develop; in addition, the value of $t_{s}$ may be established unambiguously in this way. Consequently, in the present study the Eulerian solution at $t=t_{o}$ was used to initiate a calculation in the Lagrangian coordinate system which was then carried out until $t=t_{s}$.

In the Eulerian description, the boundary-layer equations are

$$
\frac{\partial u}{\partial t}+u \frac{\partial u}{\partial x}+v \frac{\partial u}{\partial y}=U_{e} \frac{\mathrm{d} U_{e}}{\mathrm{~d} x}+\frac{\partial^{2} u}{\partial y^{2}}, \quad \frac{\partial u}{\partial x}+\frac{\partial v}{\partial y}=0,
$$

where $y$ and $v$ are the boundary-layer scaled normal coordinate and velocity (with respect to $R e^{1 / 2}$ ) and $y=0$ defines the surface. In the cylinder problem, the radius $a$ and translational speed $U_{o}$ are used to define dimensionless variables; for the vortex problem, the corresponding quantities are the distance of the vortex from the wall $a$ and the flow speed at infinity $(1-\alpha) U_{o}$, respectively. For the cylinder, $x=0$ denotes the frontal inviscid stagnation point with $x$ measuring arclength in the clockwise direction. For the vortex problem, $x=0$ denotes the location of the vortex centre for all $t$ in a frame of reference convecting uniformly with the vortex.

To resolve the sharp temporal variations associated with the impulsive start, it is convenient to introduce Rayleigh variables $\zeta$ and $\Psi$ defined by

$$
\zeta=\frac{y}{2 t^{1 / 2}}, \quad \Psi(x, \zeta, t)=\frac{\psi}{2 t^{1 / 2}},
$$

where $\psi$ is the stream function. The governing equations (3) become

$$
4 t \frac{\partial u}{\partial t}=\frac{\partial^{2} u}{\partial \zeta^{2}}+2 \zeta \frac{\partial u}{\partial \zeta}+4 t\left[\frac{\partial \Psi}{\partial x} \frac{\partial u}{\partial \zeta}-u \frac{\partial u}{\partial x}+U_{e} \frac{\mathrm{d} U_{e}}{\mathrm{~d} x}\right], \quad \frac{\partial \Psi}{\partial \zeta}=u
$$

and the associated conditions at the surface and mainstream require that

$$
u=-\beta, \quad \Psi=0 \quad \text { at } \quad \zeta=0 ; \quad u \rightarrow U_{e} \quad \text { as } \quad \zeta \rightarrow \infty .
$$

The initial conditions are obtained by letting $t \rightarrow 0$ in equations (5) and the solutions satisfying conditions (6) are

$$
\begin{gathered}
u=\left(U_{e}+\beta\right) \operatorname{erf}(\zeta)-\beta, \\
\Psi=\left(U_{e}+\beta\right)\left[\zeta \operatorname{erf}(\zeta)-\frac{1}{\pi^{1 / 2}}\left(1-\mathrm{e}^{-\zeta^{2}}\right)\right]-\beta \zeta .
\end{gathered}
$$


For the cylinder problem the streamwise boundary conditions are periodicity in $x$, while in the vortex problem the boundary layer at infinity is not influenced by the vortex; in the latter case, the solution is also given by equations (7) for all $t$.

It is convenient to introduce computational spatial coordinates $(\widehat{x}, \widehat{\zeta})$ according to

$$
x=h_{x}(\widehat{x}), \quad 0 \leqslant \widehat{x} \leqslant 1 ; \quad \zeta=k_{\zeta} \tan \left(\frac{1}{2} \pi \widehat{\zeta}\right), \quad 0 \leqslant \widehat{\zeta} \leqslant 1,
$$

in order that calculations are carried out within a finite domain. The streamwise transformations $h_{x}$ that were utilized will be discussed subsequently. For the normal coordinate, the constant $k_{\zeta}$ controls the distribution of mesh points, with smaller values of $k_{\zeta}$ corresponding to a finer resolution near the wall (in terms of the physical coordinate $\zeta$ ). In computational coordinates, the governing equations are of the form

$$
\frac{\partial u}{\partial t}=T \frac{\partial^{2} u}{\partial \widehat{\zeta}^{2}}+P \frac{\partial u}{\partial \widehat{\zeta}}+Q \frac{\partial u}{\partial \widehat{x}}+\Gamma, \quad \frac{\partial \Psi}{\partial \widehat{\zeta}}=\frac{\pi k_{\zeta}}{2} \sec ^{2}\left(\frac{1}{2} \pi \widehat{\zeta}\right) u
$$

where the functional coefficients $P, Q, T$ and $\Gamma$ are listed in Appendix A. A uniform mesh in computational coordinates $(\widehat{x}, \widehat{\zeta})$ was adopted, and the numerical method used is similar to that described by Peridier et al. (1991). The scheme for equation $(9 a)$ is second-order accurate in both space and time and is an alternating-directionimplicit (ADI) method; at fixed $t$, integration of equation $(9 b)$ was accomplished using a form of Simpson's rule. The Eulerian calculations were carried out to $t=t_{o}$ and then continued in the Lagrangian formulation.

The unsteady Lagrangian boundary-layer equations are given by (Van Dommelen \& Shen, 1980; Peridier et al. 1991):

$$
\begin{aligned}
\frac{\partial u}{\partial t}=U_{e} \frac{\mathrm{d} U_{e}}{\mathrm{~d} x}+ & {\left[\frac{\partial x}{\partial \xi} \frac{\partial}{\partial \eta}-\frac{\partial x}{\partial \eta} \frac{\partial}{\partial \xi}\right]^{2} u, \quad \frac{\partial x}{\partial t}=u, } \\
& \frac{\partial x}{\partial \xi} \frac{\partial y}{\partial \eta}-\frac{\partial x}{\partial \eta} \frac{\partial y}{\partial \xi}=1,
\end{aligned}
$$

where equations (10) and (11) are the momentum and continuity equations, respectively. In the Lagrangian formulation, $(x, y)$ are independent variables and $(\xi, \eta)$ are the dependent coordinates representing the initial location of the particles at $t=t_{0}$; hence the initial conditions are given by

$$
x=\xi, \quad y=\eta \quad \text { at } \quad t=t_{o},
$$

along with a prescribed streamwise velocity field $u(\xi, \eta)$ at $t=t_{o}$. A key feature of equations (10) is that they do not involve the normal coordinate $y$ or velocity $v$; it is these quantities which increase rapidly and develop sharp streamwise variations as separation is approached. Eventually, such behaviour severely impacts the viability of an Eulerian approach. On the other hand, the streamwise particle positions $x(\xi, \eta, t)$ and velocities $u(\xi, \eta, t)$ remain regular even at separation, and this is one of the principal advantages of using the Lagrangian formulation. The curves $x=$ constant denote the current streamwise location (at time $t$ ) of particles which originated on a line of constant $x$ (at the initial time $t$ ); these curves are characteristics of the continuity equation (11) which has the following integral:

$$
y=\int_{0}^{s} \frac{\mathrm{d} s}{\left[(\partial x / \partial \xi)^{2}+(\partial x / \partial \eta)^{2}\right]^{1 / 2}},
$$

where $s$ is the distance from the wall measured along the characteristic $x=$ constant. 
This relation gives the normal coordinates $y(\xi, \eta, t)$ in terms of the calculated $x(\xi, \eta, t)$ field. The continuity equation, as expressed by (13), provides an unambiguous criterion to determine the instant and location of unsteady separation, namely the first instant when both $\partial x / \partial \xi$ and $\partial x / \partial \eta$ vanish simultaneously at some location $\left(\xi_{s}, \eta_{s}\right)$. Because the momentum and continuity equation are decoupled, it is only necessary to solve the system (10) and test at each time step for the first appearance of a stationary point (i.e. $\partial x / \partial \xi=\partial x / \partial \eta=0$ ). At this time $t_{s}$, the calculations must be terminated. An algorithm to determine $\left(\xi_{s}, \eta_{s}\right)$ and $t_{s}$ is described in Appendix B; the method involves tracking the two curves $\partial x / \partial \xi=0$ and $\partial x / \partial \eta=0$ and determining the instant and location where they first touch. The evolution of a stationary point implies (from equation (13)) that a fluid particle originally at $\left(\xi_{s}, \eta_{s}\right)$ has been compressed to zero streamwise thickness and a portion is now at an infinite distance from the wall on the boundary-layer scale (Cowley et al. 1990). For any time $t<t_{s}$, the Eulerian flow field may be constructed for fixed $t$ by integration of equation (13) (Peridier et al. 1991).

In Lagrangian coordinates, the boundary conditions for both problems are

$$
u=-\beta \quad \text { at } \eta=0 ; \quad u \rightarrow U_{e}(x) \quad \text { as } \quad \eta \rightarrow \infty .
$$

For the cylinder problem, the solution must be periodic in $\xi$. For the vortex problem, it may be inferred from equation (2) that the boundary-layer motion at infinity is a plane-parallel flow; thus $y=\eta$ for all time and from equation (7a)

$$
u=\left(U_{e}+\beta\right) \operatorname{erf}\left[\frac{\eta}{2 t^{1 / 2}}\right]-\beta \quad \text { as } \xi \rightarrow \pm \infty .
$$

Again, independent computational coordinates $(\widehat{\xi}, \widehat{\eta})$ are defined on a finite domain according to

$$
\xi=h_{\xi}(\widehat{\xi}), \quad 0 \leqslant \widehat{\xi} \leqslant 1 ; \quad \eta=k_{\eta} \tan \left(\frac{1}{2} \pi \widehat{\eta}\right), \quad 0 \leqslant \widehat{\eta} \leqslant 1,
$$

with dependent computational coordinates $(\widehat{x}, \widehat{y})$ given by

$$
x=h_{x}(\widehat{x}), \quad 0 \leqslant \widehat{x} \leqslant 1 ; \quad y=k_{y} \tan \left(\frac{1}{2} \pi \widehat{y}\right), \quad 0 \leqslant \widehat{y} \leqslant 1 .
$$

Here $k_{\eta}$ and $k_{y}$ are stretching factors for the normal coordinate. A number of transformations were considered for $\xi$ and $x$ and these will be discussed subsequently. Upon introducing (16) and (17a) into the momentum equation (10a), it may be shown that

$$
\frac{\partial u}{\partial t}=R \frac{\partial^{2} u}{\partial \widehat{\xi}^{2}}+S \frac{\partial^{2} u}{\partial \widehat{\xi} \partial \widehat{\eta}}+T \frac{\partial^{2} u}{\partial \widehat{\eta}^{2}}+P \frac{\partial u}{\partial \widehat{\eta}}+Q \frac{\partial u}{\partial \widehat{\xi}}+\Gamma,
$$

where the coefficients $P, Q, R, S, T$ and $\Gamma$ are given in Appendix A. The numerical scheme used to solve (18) is similar to that used to solve (11a) (Peridier et al. 1991). Substituting (16) and (17) into (11), the continuity equation in computational coordinates becomes

$$
\frac{\partial \widehat{x}}{\partial \widehat{\xi}} \frac{\partial \widehat{y}}{\partial \widehat{\eta}}-\frac{\partial \widehat{x}}{\partial \widehat{\eta}} \frac{\partial \widehat{y}}{\partial \widehat{\xi}}=\frac{h_{\xi}^{\prime}}{h_{x}^{\prime}} \frac{k_{\eta}}{k_{y}} \frac{\cos ^{2}\left(\frac{1}{2} \pi \widehat{y}\right)}{\cos ^{2}\left(\frac{1}{2} \pi \widehat{\eta}\right)}
$$

where the primes denote differentiation with respect to the argument.

It now remains to describe the manner in which the Lagrangian calculations are initiated from the Eulerian solution at $t=t_{o}$. If it is insisted that the mesh for the Lagrangian and Eulerian calculations be the same, then

$$
\widehat{\xi}=\widehat{x}, \quad \widehat{\eta}=\widehat{\zeta} \quad \text { at } \quad t=t_{o} .
$$


This choice is convenient because it circumvents the need to interpolate the Eulerian solution in order to obtain initial conditions for the Lagrangian calculations. Condition $(20 a)$ is easily established by requiring the transformations in $(8 a)$ and $(16 a)$ be identical. Using equations $(4 a),(12 b),(8 b)$ and $(16 b)$, it is easily shown that condition (20b) may be satisfied if the expansion factor $k_{\eta}$ for the Lagrangian calculation is chosen according to

$$
k_{\eta}=2 t_{o}^{1 / 2} k_{\zeta} .
$$

In this manner, the computed Eulerian solution for the streamwise velocity $u$ at $t_{o}$ may be used directly as the initial condition for the Lagrangian calculations.

\section{Remeshing algorithm}

Although the Lagrangian formulation allows accurate calculation of unsteady separation and provides an unambiguous criterion for its occurrence, there is an associated disadvantage which is due to what may be termed 'mesh distortion'. This difficulty is related to the rapid separation and distortion of adjacent particles which can occur as the boundary-layer flow evolves; this can result in the coefficients in equation (18) becoming large as $t$ increases, in which case the system (18) is said to become 'increasingly stiff with time'. Significant streamwise separation of initially adjacent fluid particles is generally most rapid in regions of favourable pressure gradient and by contrast much less pronounced in an adverse pressure gradient. Consider, for example, fluid particles near the boundary-layer edge close to a stagnation point for which $\partial x / \partial t \approx a x$, where $a$ is a constant; the solution for the trajectories for $x>0$ satisfying equation $(12 a)$ is

$$
x=\xi \mathrm{e}^{a\left(t-t_{o}\right)}, \quad t>t_{o},
$$

where $x=\xi$ at $t=t_{0}$. At the rear stagnation point for the cylinder problem and for $x \gtrsim-\sqrt{3}$ for the vortex problem, the pressure gradient is adverse $(a<0)$, and from equation (22) fluid particles remain in the vicinity of the stagnation point. On the other hand, near $x=0$ and for $x \lesssim \sqrt{3}$ for the cylinder and vortex problems, respectively, the pressure gradient is favourable $(a>0)$ and adjacent fluid particles are eventually separated by a distance which increases exponentially with time. Increasing stiffness of the momentum equation causes an increase in the number of iterations per time step and can eventually lead to highly inaccurate results. It is important to emphasize, however, that the reasons giving rise to convergence difficulties in the Eulerian and Lagrangian calculations are quite different. In Eulerian calculations, the onset of separation leads to the development of sharp streamwise gradients over a region of increasingly narrowing extent within zones of adverse pressure gradient; this behaviour causes an abrupt increase in the number of iterations per time step. The difficulty may be alleviated temporarily by decreasing the time step, but eventually a point in time will be reached where the solution will fail to converge at some $t<t_{s}$. On the other hand, for Lagrangian calculations, the number of iterations per time step increases gradually during the course of the integration; this behaviour is directly associated with the increasing stiffness of equation (18), which is generally associated with a region or regions of favourable pressure gradient. This is a serious problem in the Lagrangian formulation that must be overcome whenever the mainstream contains regions of favourable pressure gradient. One possible approach is to use the traditional Eulerian formulation (which is well-suited for favourable pressure gradient flows), in the initial stages of development and up until a time $t_{o}$ just before 
sharp streamwise gradients occur. In this manner, the time interval over which the Lagrangian calculation scheme is used is reduced from $t_{s}$ to $t_{s}-t_{o}$; consequently, the relative stiffness of the equations as $t$ increases is at least reduced. Unfortunately, the 'stiffness problem' is not necessarily eliminated because the time interval $t_{s}-t_{o}$ cannot be made too small; otherwise, the Eulerian solution may become corrupted by insufficient resolution for $t<t_{o}$ and hence the initial condition at $t_{o}$ for the Lagrangian will be incorrect. In general, it is not possible to determine an appropriate switchover time $t_{o}$ a priori. The value is problem dependent and typically several values of $t_{o}$ must be selected; furthermore, it must be confirmed that the calculated results from the Lagrangian coordinate formulation are independent of the choice of $t_{o}$.

In most problems, and indeed in those considered here, the time interval $t_{s}-t_{o}$ is still sufficiently large so that the increasing stiffness of the momentum equation in the Lagrangian coordinate formulation must be addressed in order to obtain accurate results. Two strategies have been adopted in the past to alleviate this difficulty. In the calculation of the boundary-layer flow past an impulsively started cylinder, Van Dommelen \& Shen $(1980,1982)$ used a transformation, which provided a finer mesh in the favourable pressure gradient region in physical space, effectively reducing the initial distance between adjacent particles there; this scheme was later adopted by Shen \& Wu (1990). Although effective, the scheme has the disadvantage that more computational effort is expended in a region of the flow where the solution is relatively well-behaved. In the study by Peridier et al. (1991), the initial flow development was calculated in the Eulerian coordinate formulation. Although several values of $t_{o}$ were considered to confirm the invariancy of the results, the value $t_{o}=0.25$ was chosen to be most convenient because this obviated the necessity of interpolating the Eulerian solution to obtain the initial conditions for the subsequent Lagrangian calculations; in effect, both expansion factors $k_{\zeta}$ and $k_{\eta}$ were assumed unity (for reference, see equations (8b), (16b) and (21). Since $t_{s}=0.989$ in the study of Peridier et al. (1991), the time interval $t_{s}-t_{o}$ was still large so that the increasing stiffness of the momentum equation had to be addressed. This was dealt with by reducing the time step with increasing time to very small values. Once again, although the strategy was effective, a substantial increase in computational time resulted, and the course of the integration slowed down drastically.

A secondary objective in this study was to develop a remeshing procedure to overcome the 'stiffness problem' in the Lagrangian formulation; such a procedure was recommended by Shen \& Wu (1990) but not implemented. In the present remeshing scheme, Lagrangian calculations were carried out for a predetermined period of time denoted by $\Delta t$. At the end of this period, the continuity equation was solved to construct the Eulerian description of the flow and, in particular, the solution for $u(x, y)$ at fixed points on a rectangular mesh. Using the initial conditions (12) with $t_{o}$ replaced by $t_{1}=t_{o}+\Delta t$ and the solution $u(x, y)$ at $t=t_{1}$, the calculations were initiated once again to perform a second Lagrangian cycle. The $k$ th cycle ended at $t_{o}+k \Delta t$, and the sequence of cycles was continued until an unsteady separation singularity was encountered. Although the mesh distorts during each cycle, remeshing occurred before the equations become excessively stiff and a reduction in the time step was required. Consequently, this scheme obviates the necessity of finer resolution in a favourable pressure gradient region or the need for smaller time steps as the calculation proceeds further in time. In using this approach, the number of solution remeshings should be kept to a minimum since the process is computationally intensive. Most importantly, however, a sufficient time interval must be allowed between the final remeshing and the time of unsteady separation $t_{s}$; this is because interpolation is required to construct 
the Eulerian flow solution and, in order to accurately carry this out, the Eulerian field must be sufficiently smooth at the time of remeshing.

The transition from one cycle to the next through the solution of the continuity equation (19) is now described. In certain situations encountered in the present study, it became necessary to adopt a different mesh for the Lagrangian calculations in the subsequent cycle; the algorithm developed here allows this added flexibility. Let the mesh that is to be used in the next calculation cycle be denoted by $\left(\xi^{*}, \eta^{*}\right)$, which is related to the corresponding computational coordinates $\left(\widehat{\xi}^{*}, \widehat{\eta}^{*}\right)$ through

$$
\xi^{*}=h_{\xi^{*}}\left(\widehat{\xi}^{*}\right), \quad 0 \leqslant \widehat{\xi}^{*} \leqslant 1 ; \quad \eta^{*}=k_{\eta}^{*} \tan \left(\frac{1}{2} \pi \widehat{\eta}^{*}\right), \quad 0 \leqslant \eta \leqslant 1 .
$$

In order to construct the initial conditions for each subsequent cycle, the streamwise velocity must be calculated at the mesh points at $\left(\widehat{\xi}^{*}, \widehat{\eta}^{*}\right)$ from the Lagrangian solution at the end of the current cycle. Suppose that the velocity is desired along a particular mesh line denoted by $\xi^{*}=\xi_{i}^{*}$ at the start of a new cycle; this line corresponds to the characteristic curve $x=x_{i}=\xi_{i}^{*}$ in the Lagrangian solution at the end of the previous cycle. Alternatively, the characteristic is given by $\widehat{x}=$ $\widehat{x}_{i}=h_{x}^{-1}\left(h_{\xi^{*}}\left(\xi_{i}^{*}\right)\right)$ in computational coordinates where the superscript -1 denotes the inverse transformation. Consequently, determining the functional dependence of $u$ on the normal coordinate $\widehat{\eta}^{*}$ at $\widehat{\xi}^{*}=\widehat{\xi}_{i}^{*}$ is equivalent to determining the variation of $u$ along the characteristic $\widehat{x}=\widehat{x}_{i}$ in the current Lagrangian solution.

Next consider the normal coordinate $\eta$. At the beginning of the Lagrangian integration, $y$ is defined by equation (12b), and at the start of each new cycle by $y=\eta^{*}$. Throughout the course of the integrations, $\widehat{y}$ and $\hat{\eta}$ are defined on a fixed rectangular grid, and physical values of $y$ and $\eta$ at any stage can be found from equations (16b) and $(17 b)$. It is convenient to take $\widehat{y}=\widehat{\eta}$ at the beginning of the Lagrangian integrations, and $\widehat{y}=\widehat{\eta}^{*}$ at the start of each new remeshing cycle. The normal expansion factor $k_{\eta}$ may be changed for the subsequent cycle; denoting this by $k_{\eta}^{*}, k_{y}$ must then be selected equal to $k_{\eta}^{*}$. Determination of the velocity $u$ at a particular value of $\widehat{\eta}^{*}=\widehat{\eta}_{j}^{*}$, say, corresponds then to finding the velocity at a location $\widehat{y}_{j}=\widehat{\eta}_{j}^{*}$ from the Lagrangian solution at the end of the current cycle along the characteristic $\widehat{x}=\widehat{x}_{i}$. The method used is an inverse characteristic scheme which is described next.

The characteristics of equation (19) are given by

$$
\frac{\mathrm{d} \widehat{\xi}}{A}=\frac{\mathrm{d} \widehat{\eta}}{B}=\frac{\mathrm{d} \widehat{y}}{C}=\mathrm{d} \widehat{s}
$$

where

$$
A=-\frac{\partial \widehat{x}}{\partial \widehat{\eta}} ; \quad B=\frac{\partial \widehat{x}}{\partial \widehat{\xi}} ; \quad C=\frac{h_{\xi}^{\prime}(\widehat{\xi})}{h_{x}^{\prime}(\widehat{\xi})} \frac{\left.k_{\eta}\right)}{k_{y}} \frac{\cos ^{2}\left(\frac{1}{2} \pi \widehat{y}\right)}{\cos ^{2}\left(\frac{1}{2} \pi \widehat{\eta}\right)},
$$

and $\mathrm{d} \widehat{s}$ is the differential arclength along the characteristic in computational coordinates. The integrations are initiated at the wall where $\hat{y}=\widehat{\eta}=0$. On the wall, the distribution of $x$ at the end of the $k$ th cycle may be evaluated from equations (10b) and $(14 a)$ and it follows that $x=\xi-\beta \Delta$ t. In terms of computational coordinates

$$
\widehat{\xi}=h_{\xi}^{-1}\left\{h_{x}(\widehat{x})+\beta \Delta t\right\} .
$$

Using this relation, an integration may be initiated for a given value of $\widehat{x}$ up the characteristic starting from $\hat{\eta}=0$ using the Lagrangian solution at the end of the current cycle. Let $\left(\widehat{\xi}_{n}, \widehat{\eta}_{n}\right)$ denote some point along the characteristic $\widehat{x}=\widehat{x}_{i}$ having an associated value of $\widehat{y}$ denoted by $\hat{y}_{n}$. To determine the coordinates of the next point 
along the characteristic $\left(\widehat{\xi}_{n+1}, \widehat{\eta}_{\bar{n} 1}\right)$ and the corresponding value of $\widehat{y}=\widehat{y}_{n+1}$, equations (24) may be discretized by writing

$$
\Delta \widehat{\xi}=A \Delta \widehat{s}, \quad \Delta \widehat{\eta}=B \Delta \widehat{s}, \quad \Delta \widehat{y}=C \Delta \widehat{s},
$$

where

$$
\Delta \widehat{\xi}=\widehat{\xi}_{n+1}-\widehat{\xi}_{n}, \quad \Delta \widehat{\eta}=\widehat{\eta}_{n+1}-\widehat{\eta}_{n}, \quad \Delta \widehat{y}=\widehat{y}_{n+1}-\widehat{y}_{n} .
$$

A predictor-corrector algorithm, which is second-order accurate in $\Delta \widehat{s}$ and is similar to that described by Peridier et al. (1991), was used to solve equations (27) and (28). Instead of specifying $\Delta \widehat{s}$ (the step length along the characteristic), the value of $\Delta \widehat{y}$ was prescribed; a number of methods of specifying $\Delta \widehat{y}$ were explored, but eventually the simple choice $\Delta \widehat{y}=\Delta h / 4$ was used throughout, where $\Delta h$ denotes the mesh size in the $\hat{y}$-direction. The basic idea here is to select the interval in $\Delta \widehat{y}$ so that the integration along the characteristic $\widehat{x}=\widehat{x}_{i}$ passes through a mesh point $\widehat{y}=\widehat{y}_{j}$ exactly (every 4 steps). In the predictor phase of the algorithm, the coefficients $A, B, C$ are evaluated at $\left(\xi_{n}, \eta_{n}\right)$ and with $\Delta \widehat{y}$ specified, $\Delta \widehat{s}$ is determined from equation $(27 c) ; \Delta \widehat{\xi}$ and $\Delta \widehat{\eta}$ are then found from equations (27a) and (27b). In the corrector phase of the algorithm, average values of $A, B$ and $C$ are evaluated midway through the interval.

The solution algorithm described here is an inverse method of characteristics and involves the determination of the initial location $\left(\widehat{\xi}_{k}, \widehat{\eta}_{k}\right)$ of the particle which is currently at the position $\left(\widehat{x}_{i}, \widehat{y}_{j}\right)$. The streamwise velocity $u$ at $\left(\widehat{x}_{i}, \widehat{y}_{j}\right)$ is obtained by using bilinear interpolation to determine $u$ at $\left(\widehat{\xi}_{k}, \widehat{\eta}_{k}\right)$ from the current computed Lagrangian solution. In this manner, the streamwise velocity is obtained on a rectangular mesh, and this is suitable to initiate the Lagrangian calculations in the subsequent cycle. The remeshing algorithm not only incorporates the added flexibility of allowing a different mesh for the subsequent cycle, but also places no restriction on a possible change in the number of streamwise and normal mesh points. In addition, the above procedure is also used in this study to obtain a Eulerian description of the flow field at any instant of time (e.g. to obtain instantaneous streamlines) from that calculated in the Lagrangian formulation.

\section{Results for the cylinder}

To test the present algorithms, the boundary-layer development was calculated for the non-rotating case $\beta=0$ and for $\beta=1.0,1.5$ to compare with previous results obtained by other authors. For $\beta=0$, two different approaches were used. For method I, Lagrangian coordinates were used from the impulsive start at $t=0$, followed by remeshing at $t=0.5$ and 1.0. In method II, the calculation was initiated in Eulerian coordinates and switched to the Lagrangian formulation at $t=1.3$. The calculations based on method II are expected to be more accurate since an explicit transformation to eliminate the irregular behaviour associated with the impulsive start was not introduced in the Lagrangian formulation for method I. A number of mesh sizes were used and the quoted results are based on a $201 \times 101$ mesh (in the streamwise and normal directions, respectively) with uniform time steps of $\Delta t=10^{-3}$ and $5 \times 10^{-4}$ for the Eulerian and Lagrangian calculations respectively. The mesh distribution in the streamwise direction is uniform in the sense that $h_{x}=2 \pi \widehat{x}$ and $h_{\xi}=2 \pi \widehat{\xi}$ in equations $(8 a),(17 a)$ and $(16 a)$, respectively.

Calculated results are shown in table 1 , where $x_{s}$ and $t_{s}$ are the location and time of unsteady separation, respectively, and $u_{s}$ is the velocity of the particle at separation. 


\begin{tabular}{|c|c|c|c|c|c|c|}
\hline \multirow[b]{3}{*}{ Method I } & \multicolumn{3}{|c|}{$\beta=0$} & \multicolumn{3}{|c|}{$\beta=1.5$} \\
\hline & $x_{s}$ & $t_{s}$ & $u_{s}$ & $x_{s}$ & $t_{s}$ & $u_{s}$ \\
\hline & $1.934,4.349$ & 1.500 & $-0.535,0.535$ & 3.486 & 1.769 & 0.05 \\
\hline Method II & $1.937,4.346$ & 1.503 & $-0.533,0.533$ & 3.483 & 1.765 & 0.066 \\
\hline Van Dommelen (1981) & $1.937,4.346$ & 1.502 & 0.52 & - & - & - \\
\hline Wu (1991) & - & - & - & 3.478 & 1.77 & 0.06 \\
\hline
\end{tabular}

Although the flow for $\beta=0$ is symmetric, this condition was not explicitly introduced into the numerical procedure; consequently, the unsteady separation singularity occurs simultaneously on the upper and lower halves of the cylinder. The results agree well with one another and with the calculations of Van Dommelen (1981)†, who used an effective spatial mesh of $(579 \times 129)$. Calculations were also carried out using method II with switchover times of $t_{o}=0.25$ and 1.0; the values of $x_{s}$ and $t_{s}$ agree with those in table 1 up to three significant digits; however, only two significant digit accuracy agreement was found for $u_{s}$. This is typical for Lagrangian calculations, and as noted by Van Dommelen (1981), it is hardest to obtain grid-independent results for $u_{s}$.

The second test case considered was for $\beta=1.0$, 1.5, which has recently been calculated by Shen \& Wu (1990) and Wu (1991) on a $(65 \times 65)$ mesh. For method I, an additional remeshing at $t_{2}=1.5$ was performed, and, for method II, the switch over was implemented at $t_{o}=1.0$ with a remesh at $t_{1}=1.5$. The time steps and mesh were the same as for $\beta=0$. The calculated results are in good agreement with those obtained by Shen \& Wu (1990) and $\mathrm{Wu}(1991)$ as seen in table 1 for $\beta=1.5$. It is evident that the present remeshing algorithm yields good accuracy and is an effective cure for the 'stiffness' problem in the Lagrangian formulation.

The maximum value of $\beta$ considered by Wu (1991) is $\beta=1.5$; in the present study the objective is to consider larger values of $\beta$ to determine the critical value $\beta_{c}$ beyond which unsteady separation is suppressed. To this end, solutions for values of $\beta$ in the range 1.5-2.0 were calculated. Initially, the two methods described for $\beta=1.5$ were also used for larger values of $\beta$. However, as the value of $\beta$ increased, a uniform mesh in $\xi$ was found to be increasingly unappealing because it yielded insufficient resolution to accurately determine the instant of separation. The reason may be appreciated from figure 4 which shows the curves $\partial \widehat{x} / \partial \widehat{\xi}=0$ and $\partial \widehat{x} / \partial \widehat{\eta}=0$ at the instant of separation for $\beta=1.60$ and $\beta=1.80$. There are two curves $\partial \widehat{x} / \partial \widehat{\eta}=0$, which are both attached to the cylinder and appear at a fairly early stage in the calculations. The curves $\partial \widehat{x} / \partial \widehat{\xi}=0$ appear at a much later stage as closed curves in the middle of the boundary layer. Separation corresponds to the first instant when the curves $\partial \widehat{x} / \partial \widehat{\eta}=0$ and $\partial \widehat{x} / \partial \widehat{\xi}=0$ touch. It may be noted from figure 4 that this condition is achieved in the third quadrant but by $t=t_{s}$ is not close to occurring anywhere in the upper half-plane $(\widehat{\xi}<0.5)$. In fact as shown in figures $4(a)$ and $4(b)$, the curves $\partial \widehat{x} / \partial \widehat{\xi}=0$ and $\partial \widehat{x} / \partial \widehat{\eta}=0$ draw farther apart in the upper-half plane as the rotation rate increases. The streamwise extent of the relevant curve at separation, $\partial \widehat{x} / \partial \widehat{\xi}=0$ in figure $4(a)$, is sufficiently large and is well resolved by the uniform

$\dagger$ Van Dommelen (1981) non-dimensionalized the governing equations using twice the translational speed of the cylinder. Consequently, the time and velocity given in table 1 are numerically one-half and twice, respectively, of those reported by Van Dommelen. 

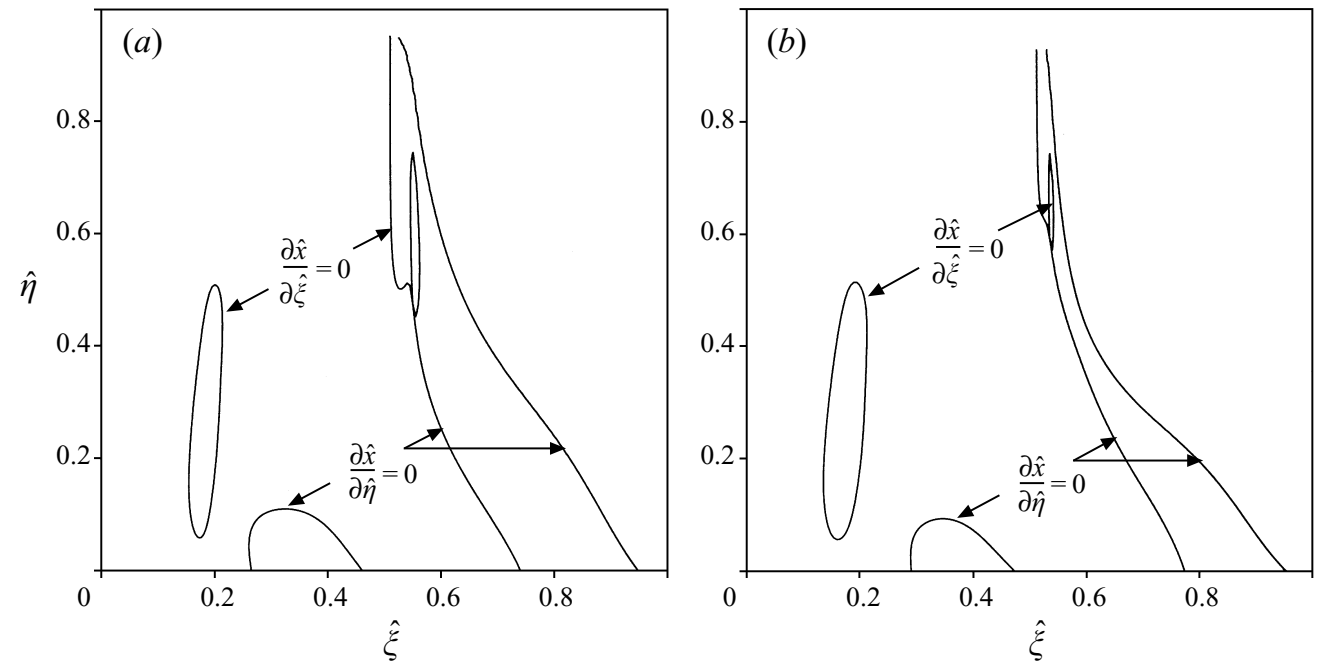

Figure 4. Contours of $\partial \widehat{x} / \partial \widehat{\xi}=0$ and $\partial \widehat{x} / \partial \widehat{\eta}=0$ at separation $t=t_{s}$ for (a) $\beta=1.6$ and (b) $\beta=1.8$.

mesh; however, as $\beta$ increases, the streamwise extent of the curve $\partial \widehat{x} / \partial \widehat{\xi}=0$ becomes increasingly narrow, as typified in figure $4(b)$. The lower tip of the curve $\partial \widehat{x} / \partial \widehat{\xi}=0$ is increasingly sharp as $\beta$ increases, and due to a lack of resolution near the sharp tip, it is difficult to determine accurately the instant when the curves first touch. To overcome this problem, the calculations beyond the remeshing at $t_{1}=1.5$ were carried out using the following transformation in the streamwise direction:

$$
\xi=h_{\xi}(\widehat{\xi})=\pi\left\{1+\frac{\tan \left[\frac{1}{2} \pi k_{\xi}(2 \widehat{\xi}-1)\right]}{\tan \left[\frac{1}{2} \pi k_{\xi}\right]}\right\} .
$$

Here $k_{\xi}$ is a parameter, and for $0<k_{\xi}<1$, relatively more streamwise mesh points are clustered near the rear inviscid stagnation point; the mesh resolution near $x=\pi$ increases with increasing $k_{\xi}$. This mesh is subsequently referred to as a non-uniform mesh. At the same time, a uniform transformation for the dependent variable $x$ is retained (i.e. $h(\widehat{x})=2 \pi \widehat{x}$ in equations $(8 a)$ and $(17 a)$ ). Using the transformation (29), the region near $x=\pi$ in physical space is expanded in computational space giving rise to an apparent tangential thickening of the relevant curve $\partial \widehat{x} / \partial \widehat{\xi}=0$ and facilitating an accurate determination of $t_{s}$. Several combinations of mesh size, time step, remeshing times and values of $k_{\xi}$ were used, and four methods are summarized in table 2, with the results given in table 3 . The values of the location, $x_{s}$, and instant, $t_{s}$, of unsteady separation in table 2 are reported to three significant digits, whereas the value of the separation velocity, $u_{s}$, is given to two digits. For the mesh systems used in this study, no unsteady separation was detected for $\beta>1.91$.

It is evident from table 3 that all methods give much the same answers for the lower values of $\beta$ for $x_{s}$ and $t_{s}$, but that an accurate estimate of $u_{s}$ is more difficult, as previously discussed. With increasing values of $\beta$, the non-uniform mesh is necessary to achieve accurate results, and the agreement between calculated results for methods III and IV gives confidence in the results. Figure 5 shows the variation of $x_{s}, t_{s}$ and $U_{e}\left(x_{s}\right)-u_{s}$ (with values taken from method IV of table 3 ) as a function of the wall speed parameter $\beta$. With increasing $\beta$, the singularity time $t_{s}$ is delayed and the 


\begin{tabular}{ll}
\hline Method & \multicolumn{1}{c}{ Description } \\
I & Impulsive start in Lagrangian coordinates. \\
& Solution remeshed at intervals of 0.5 time units. \\
& Uniform mesh $(201 \times 101) ; \Delta t=5 \times 10^{-4}$. \\
II,III,IV $\quad$ & Impulsive start in Eulerian coordinates. \\
& Uniform mesh $(201 \times 101) ; \Delta t=10^{-3}$. \\
& Switchover to Lagrangian calculations at $t_{o}=1.0$. \\
& Lagrangian calculations until $t_{1}=1.5$. \\
& Uniform mesh $(201 \times 101) ; \Delta t=5 \times 10^{-4}$. \\
& Lagrangian solution remeshed at $t_{1}=1.5$. \\
& Uniform mesh $(201 \times 101) ; \Delta t=5 \times 10^{-4}$. \\
II & Lagrangian solution remeshed at $t_{1}=1.5$. \\
& Non-uniform mesh $(201 \times 101)$ with $k_{\xi}=0.7 ; \Delta t=5 \times 10^{-4}$. \\
III & Lagrangian solution remeshed at $t_{1}=1.5$. \\
& Non-uniform mesh $(401 \times 101)$ with $k_{\xi}=0.7 ; \Delta t=2 \times 10^{-4}$. \\
IV $\quad$ &
\end{tabular}

location of the singularity $x_{s}$ shifts progressively from within the fourth quadrant toward regions of low mainstream velocity near the rear inviscid stagnation point at $x=\pi$; in the fourth quadrant the wall and mainstream velocities are in the same direction. The variation of $U_{e}\left(x_{s}\right)-u_{s}$ is of particular significance since its magnitude decreases monotonically with increasing $\beta$, possibly becoming zero just slightly beyond $\beta=1.91$. A general theory of unsteady two-dimensional separation has been discussed by Elliott et al. (1983), who argue that the boundary layer bifurcates at separation into three regions in a band which progressively narrows in the streamwise direction as the process evolves toward separation; in this structure two thin layers, one adjacent to the wall and one which grows rapidly away from the surface, bracket an expanding central inviscid region where the vorticity is almost zero. A schematic diagram of a typical velocity profile for the cylinder problem at separation is shown in figure $6(a)$, for a typical case occurring for small rotational velocities, say $\beta=\beta_{1}$. In the present problem, separation occurs in the fourth quadrant where $U_{e}(x)$ (as well as the wall velocity) is negative, and figure $6(a)$ is constructed to reflect this environment. Note that in accordance with the physical picture of separation described by Van Dommelen \& Shen $(1980,1982)$ and Elliott et al. (1983), the velocity is very flat in the central region, but adjusts rapidly through the upper and lower layers (shown by broken lines) in order to meet the mainstream and wall velocities, respectively. For the typical case at low rotation rates, the velocity in the central region $u_{s}$ is positive, and at separation, the compressed fluid particle is moving in a direction opposite to that of the mainstream. However, $u_{s}$ decreases with increasing wall speed and for a value $\beta_{2}$ (which is approximately $\beta_{2}=1.63$ for the cylinder problem), the velocity in the central region vanishes. As the wall speed increases further, the compressed fluid particle at separation has negative velocity and moves in the same direction as the mainstream. Note, however, that in all cases the wall appears to be moving downstream in a frame of reference moving with the separation, and each situation is therefore a case of upstream-slipping separation. With increasing $\beta$, the streamwise location of separation moves progressively closer to $x=\pi$ and consequently $U_{e}\left(x_{s}\right)$ decreases. Eventually, a critical value $\beta_{c}$ is reached where $U_{e}\left(x_{s}\right)-u_{s}$ vanishes (in this case just beyond $\beta=1.91$ ) at a value near to, but distinct from, $x=\pi$. 


\begin{tabular}{|c|c|c|c|c|}
\hline \multirow[b]{2}{*}{$\beta$} & \multicolumn{4}{|c|}{ Method } \\
\hline & I & II & III & IV \\
\hline 1.5 & $\begin{aligned} x_{s} & =3.48 \\
t_{s} & =1.77 \\
u_{s} & =0.049\end{aligned}$ & $\begin{array}{l}3.48 \\
1.77 \\
0.039\end{array}$ & $\begin{array}{l}3.48 \\
1.76 \\
0.066\end{array}$ & $\begin{array}{l}3.48 \\
1.76 \\
0.058\end{array}$ \\
\hline 1.55 & $\begin{array}{l}3.45 \\
1.79 \\
0.047\end{array}$ & $\begin{array}{l}3.45 \\
1.79 \\
0.036\end{array}$ & $\begin{array}{l}3.45 \\
1.79 \\
0.048\end{array}$ & $\begin{array}{l}3.45 \\
1.78 \\
0.044\end{array}$ \\
\hline 1.6 & $\begin{array}{l}3.42 \\
1.82 \\
0.044\end{array}$ & $\begin{array}{l}3.41 \\
1.82 \\
0.034\end{array}$ & $\begin{array}{l}3.41 \\
1.81 \\
0.0021\end{array}$ & $\begin{array}{l}3.41 \\
1.81 \\
0.0017\end{array}$ \\
\hline 1.65 & $\begin{array}{c}3.38 \\
1.86 \\
-0.022\end{array}$ & $\begin{array}{l}3.38 \\
1.86 \\
0.0031\end{array}$ & $\begin{array}{c}3.38 \\
1.84 \\
-0.036\end{array}$ & $\begin{array}{c}3.38 \\
1.83 \\
-0.021\end{array}$ \\
\hline 1.70 & $\begin{array}{c}3.34 \\
1.88 \\
-0.060\end{array}$ & $\begin{array}{c}3.34 \\
1.88 \\
-0.067\end{array}$ & $\begin{array}{c}3.34 \\
1.86 \\
-0.036\end{array}$ & $\begin{array}{c}3.34 \\
1.86 \\
-0.039\end{array}$ \\
\hline 1.75 & $\begin{array}{c}3.31 \\
1.90 \\
-0.074\end{array}$ & $\begin{array}{c}3.30 \\
1.90 \\
-0.079\end{array}$ & $\begin{array}{c}3.30 \\
1.89 \\
-0.079\end{array}$ & $\begin{array}{c}3.30 \\
1.89 \\
-0.082\end{array}$ \\
\hline 1.80 & $\begin{array}{c}3.27 \\
1.94 \\
-0.081\end{array}$ & $\begin{array}{c}3.27 \\
1.94 \\
-0.084\end{array}$ & $\begin{array}{r}3.27 \\
1.92 \\
-0.12\end{array}$ & $\begin{array}{r}3.27 \\
1.91 \\
-0.12\end{array}$ \\
\hline 1.85 & $\begin{array}{r}3.24 \\
1.97 \\
-0.15\end{array}$ & $\begin{array}{r}3.24 \\
1.97 \\
-0.15\end{array}$ & $\begin{array}{r}3.24 \\
1.95 \\
-0.15\end{array}$ & $\begin{array}{r}3.24 \\
1.94 \\
-0.13\end{array}$ \\
\hline 1.87 & $\begin{array}{c}3.19 \\
2.36 \\
-0.015\end{array}$ & $\begin{array}{c}3.20 \\
2.22 \\
-0.011\end{array}$ & $\begin{array}{r}3.23 \\
1.97 \\
-0.13\end{array}$ & $\begin{array}{r}3.23 \\
1.96 \\
-0.13\end{array}$ \\
\hline 1.90 & - & - & $\begin{array}{r}3.21 \\
2.02 \\
-0.11\end{array}$ & $\begin{array}{r}3.21 \\
2.00 \\
-0.12\end{array}$ \\
\hline 1.91 & - & - & $\begin{array}{r}3.20 \\
2.05 \\
-0.11\end{array}$ & $\begin{array}{r}3.20 \\
2.03 \\
-0.12\end{array}$ \\
\hline
\end{tabular}

TABLE 3. Calculated values of the location $x_{s}$, instant $t_{s}$ and speed $u_{s}$ of the unsteady separation singularity for the cylinder problem.

In the structure delineated by Van Dommelen (1981) and Elliott et al. (1983), $U_{e}\left(x_{s}\right)-u_{s}$ may be considered to be a measure of the strength of the upper separating shear layer, and thus a vanishing value of $U_{e}\left(x_{s}\right)-u_{s}$ implies a disappearance of the upper shear layer and suppression of separation. The precise value of $\beta_{c}$ is difficult to establish because a separate extensive calculation must be carried out for each value of $\beta$. However, it is evident from the present results that suppression of separation occurs at a value of $\beta_{c}=1.9$ to two significant digits. Note that this critical wall speed is slightly less than the maximum external velocity, which is 2 for the cylinder problem (at $x=\pi / 2,3 \pi / 2)$. Figure $6(b)$ shows the actual calculated velocity profiles at separation for various values of $\beta$ in the range 1.6 to 1.91 , and here the structure 


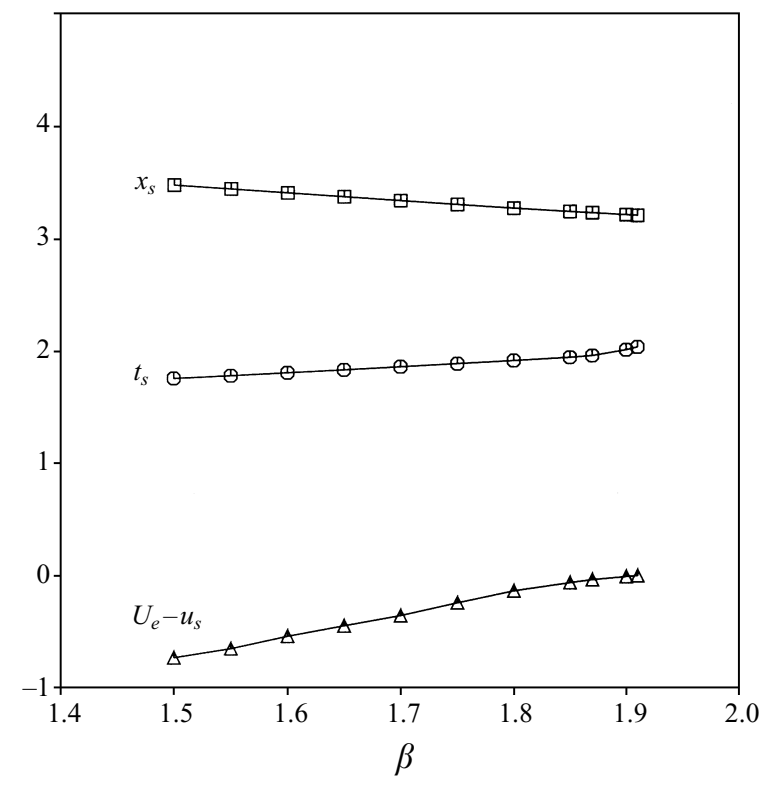

FiguRE 5. Variation of $x_{s}, t_{s}, U_{e}\left(x_{s}\right)-u_{s}$ with wall speed $\beta$.

described by Elliott et al. (1983) and shown in figure 6(a) may be clearly seen. It may be noted that all separation profiles are of the 'upstream-slipping' type (downstreammoving wall), and there is no evidence of 'downstream-slipping' (upstream-moving wall) separation.

The decreasing strength of separation with increasing $\beta$ may also be inferred from the form of the perturbation function $\widetilde{\psi}$ just prior to $t_{s}$, which is defined as

$$
\psi \sim U_{e}-\widetilde{\psi} \quad \text { as } \quad y \rightarrow \infty,
$$

where $\psi$ is the streamfunction. Note that for flows with a stationary surface, $\widetilde{\psi}=U_{e} \delta^{*}$ where $\delta^{*}$ is the displacement function; in general, $\partial \widetilde{\psi} / \partial x$ may be identified with the perturbation normal velocity associated with the developing boundary-layer flow. Figure 7 shows plots of $\widetilde{\psi}$ just prior to $t_{s}$ for various values of $\beta$ in the range 1.6-1.91. For lower values of $\beta$, a large negative spike develops in $\widetilde{\psi}$, indicating a narrow zone of strong outward normal velocity followed by a strong inflow. The characteristic spiking appears to be diminished as $\beta$ approaches $\beta_{c}$; however, it should be noted that all but one of the spikes shown in figure 7 actually decrease to minus infinity at the tip. Although figure 7 is obtained from actual computed results, the figure should be regarded as schematic in a sense, reflective of the fact that the spiking occurs in a band that becomes increasingly narrow in the streamwise direction as $\beta$ increases; it therefore becomes increasingly difficult as $\beta$ increases to perform a calculation along the precise characteristic that yields an infinite value of $\widetilde{\psi}$. As $\beta \rightarrow \beta_{c}$, the spike actually reverses and is positive, now implying two regions of strong outflow bracketing a thin zone of inflow. Although the relative magnitude of $\widetilde{\psi}$ on the upper half of the cylinder increases with $\beta$, the growth of $\widetilde{\psi}$ with time is gradual and is principally associated with the slow diffusion of vorticity from the surface over a streamwise distance $O(1)$. It is of interest to note that calculations for $\beta>1.85$ can be carried out in the Eulerian formulation with the meshes used here up to and beyond $t_{s}$ without encountering the convergence difficulties experienced at lower values of $\beta$; 

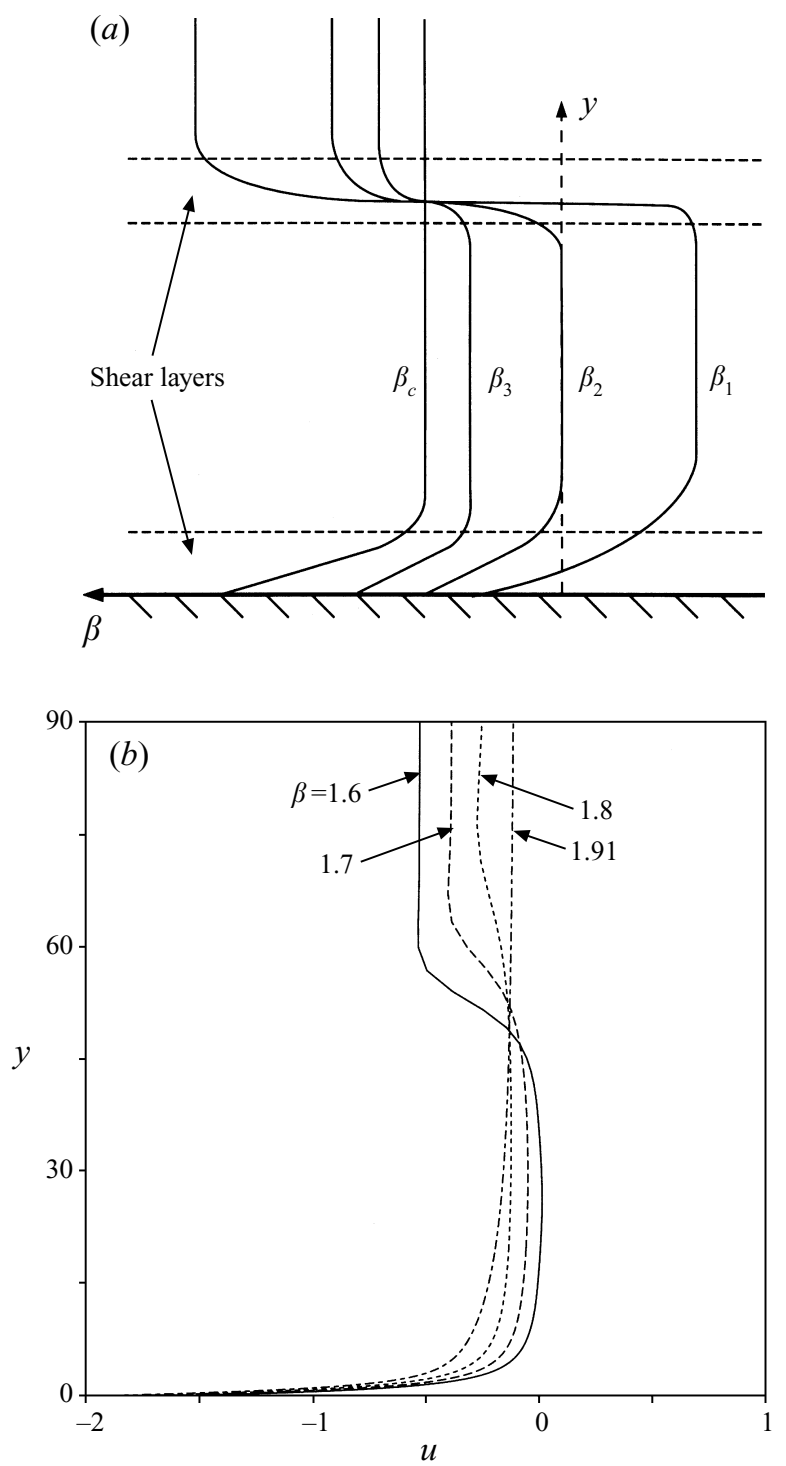

FIGURE 6. Velocity profiles at separation for increasing wall speed $\beta:(a)$ schematic diagram, (b) calculated results for the cylinder problem.

the Lagrangian calculations show that such computations are incorrect. Although it is conceivable that Eulerian computations may fail on a finer mesh, this behaviour is indicative of the relative weakness of the singularity for values of $\beta$ approaching $\beta_{c}$.

Figure 8 shows the instantaneous streamlines just prior to $t_{s}$ for $\beta=1.5,1.7$ and 1.75. The streamfunction is calculated by integrating $\partial \psi / \partial y=u$ along lines of constant $x$ once a Eulerian description of the velocity distribution is obtained by following the procedure outlined in $\S 4$. In figure $8(a)$, there are two recirculation regions above the cylinder surface: the larger one occurs above the upper surface, where the wall and mainstream velocities are in opposite directions, while the smaller one is in the fourth quadrant. Note that the wiggles near the stagnation point far from 


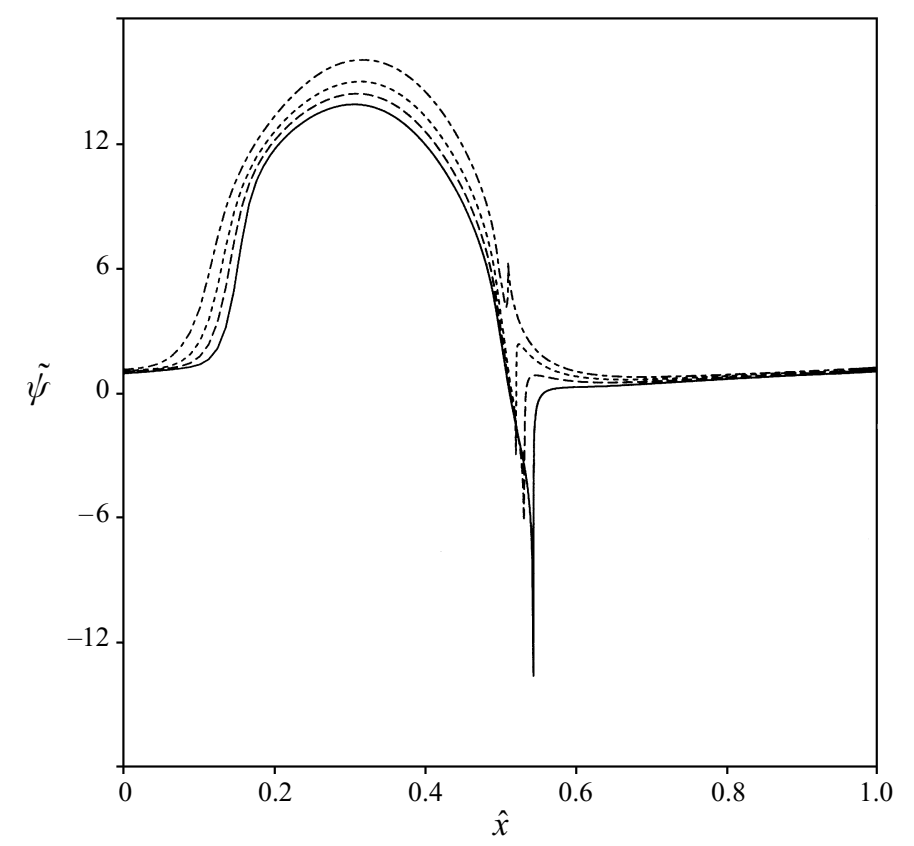

FIGURE 7. Perturbation streamfunction $\widetilde{\psi}$ at separation for various values of $\beta$ :,$- \beta=1.6$; $-\_-, \beta=1.75 ;---, \beta=1.85 ;---\_, \beta=1.91$.

the cylinder are a result of the failure of the contour plotting routine to adequately represent the streamlines near a saddle point; the actual numerical data are smooth there. The unsteady separation location is just upstream of the lower recirculation region where the streamlines may be seen to converge in a band of very narrow streamwise extent. The global features of the flow are similar to the results obtained by Ece et al. (1984). As the wall-speed parameter $\beta$ increases, the streamwise extent of the lower recirculation region diminishes as seen in figure $8(b)$ for $\beta=1.7$. As $\beta$ increases further, the lower eddy along with its associated saddle point do not form but instead a weak 'jet-like' flow pattern develops as seen in figure $8(c)$ for $\beta=1.75$. The streamline patterns are qualitatively similar to those in figure $8(c)$ for values of $\beta$ beyond 1.75 and up to the critical value $\beta_{c}$.

The vorticity $\omega$ in Lagrangian coordinates is given by

$$
\omega=-\frac{\partial x}{\partial \xi} \frac{\partial u}{\partial \eta}+\frac{\partial x}{\partial \eta} \frac{\partial u}{\partial \xi},
$$

and since separation is characterized by $\partial x / \partial \xi=\partial x / \partial \eta=0$, one of the zero vorticity lines must pass through the separation point (MRS II). The development of the topology of the zero vorticity line is therefore of some interest here. For $\beta<2$, two lines of zero vorticity are present immediately after the impulsive start, which asymptote $x=0$ and $x=\pi$ near the boundary-layer edge and which terminate on the cylinder surface. For the non-rotating case $\beta=0$, these zero vorticity lines are straight and remain so for all $t>0$ as a consequence of symmetry of the flow field; however, at a later stage the development of recirculating flow leads to the evolution of another zero vorticity line that is attached to the cylinder and intersects the zero vorticity line on the downstream radius at a point labelled A in figure $9(a)$. 

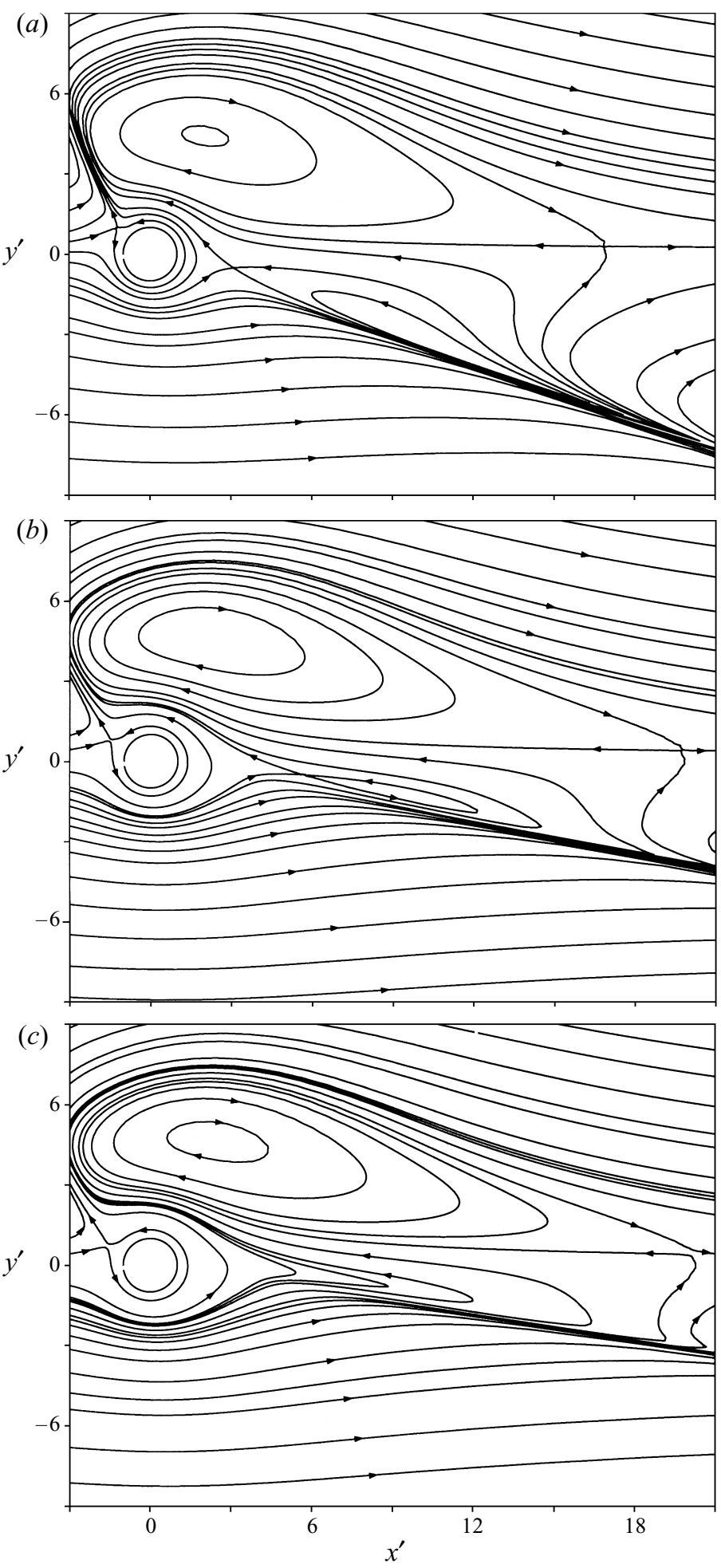

FIGURE 8. Instantaneous streamlines for the cylinder problems at separation for (a) $\beta=1.5$, (b) $\beta=1.7$, (c) $\beta=1.75 ; x^{\prime}$ and $y^{\prime}$ denote Cartesian coordinates. 
The dotted lines show schematically how and where $\omega=0$ deforms at separation in a spike on the upper and lower surfaces. When there is rotation and $\beta<2$, two $\omega=0$ lines originate on the cylinder surface in the lower half-plane (as shown in figure $9(b)$, lines B and C). Near $t_{s}$ the difference in the topologies of figures $9(a)$ and $9(b)$ is that the intersection at $\mathrm{A}$ in figure $9(a)$ has split to give rise to two curves for $\omega=0$ : (i) a line D attached to the upper surface that progressively moves away toward the second quadrant with the continued rotation of the cylinder, and (ii) a line $\mathrm{C}$ originating on the cylinder that trails outward to the boundary-layer edge eventually asymptoting to radius $\theta=\pi$. As shown schematically in figure $9(b)$, separation eventually occurs on line $\mathrm{C}$. As the rotation rate increases further, the lines $\mathrm{B}$ and $\mathrm{C}$ move progressively closer in the topology near the cylinder at the separation time, as shown in figure $9(c)$. For a sufficiently high value of $\beta$, a new configuration occurs in which lines $\mathrm{B}$ and $\mathrm{C}$ merge to form the new lines $\mathrm{F}$ attached to the cylinder and a line $\mathrm{E}$, which on the scale of the cylinder appears to originate in a narrow band emanating from large distances away. By this stage, the separation process has been weakened dramatically, and the last vestiges occur as indicated in figure $9(d)$ on line E. Figure 10 shows a plot of calculated results for $\omega=0$ for $\beta=1.8$ and 1.85 just prior to separation where the spiky behaviour of the curve $\omega=0$ may be noted, and the features discussed in connection with the schematic diagram may be confirmed. For values of $\beta=1.8$ and below, the curve $\omega=0$, which is relevant to separation, originates at the cylinder surface and passes through the separation point before approaching the boundary-layer edge (line $\mathrm{C}$ in figure $9 b$ ). On the other hand, for $\beta=1.85$ and beyond, the relevant curve $\omega=0$ that passes through the separation point appears to originate and terminate at the boundary-layer edge on the scale of the plot (line E in figure 9d). A theoretical description of the suppression of the unsteady separation is given in Appendix $\mathrm{C}$ which is consistent with the present numerical results.

Finally, it may be noted that the flow pattern in the vicinity of the saddle point located above the upper surface of the cylinder in the second quadrant (as shown in figure 8) is similar to the calculated results of Ece et al. (1984) and the inferred streamline pattern in the flow visualization studies of Koromilas \& Telionis (1980), as well as the flow structure of upstream-moving wall unsteady separation proposed by Sears \& Telionis (1975). However, in the Lagrangian coordinate formulation used here, it is evident that although this streamline pattern develops in the flow, it is not associated with unsteady separation; indeed, it may be noted that the saddle point is in a region of favourable mainstream pressure gradient and moves closer to the streamwise location $x=0$ (the frontal inviscid stagnation point) as $\beta \rightarrow \beta_{c}$. For values of $\beta>\beta_{c}$, calculations could be carried on in the boundarylayer formulation almost indefinitely with no evidence of any spiky behaviour. The large recirculation zone above the upper surface that is evident in figure 8(c) grows continuously in the normal direction with time. However, in a narrow band near the downstream radius $(x=\pi)$, an irregular behaviour in the streamline patterns eventually developed near the downstream end of the recirculation region at large times. The calculated results are suggestive of the development of an instability in the far wake, possibly of the type described by Cowley, Hocking \& Tutty (1985) (see also, Bhaskaran, Smith \& Timoshin 1995), since the sharp 'wiggles' in the streamlines did not disappear even with several reductions in the mesh sizes. An interaction with the external flow may possibly occur at large times for $\beta>\beta_{c}$, but this event is expected to be considerably less dramatic than that associated with boundary-layer separation. 


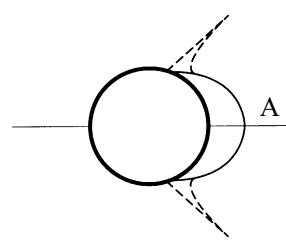

(a)

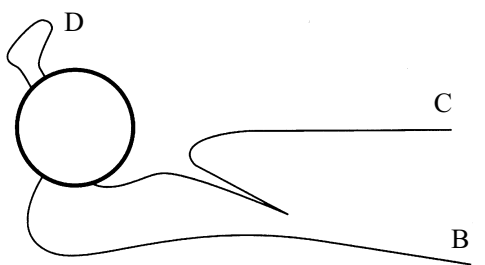

(b)
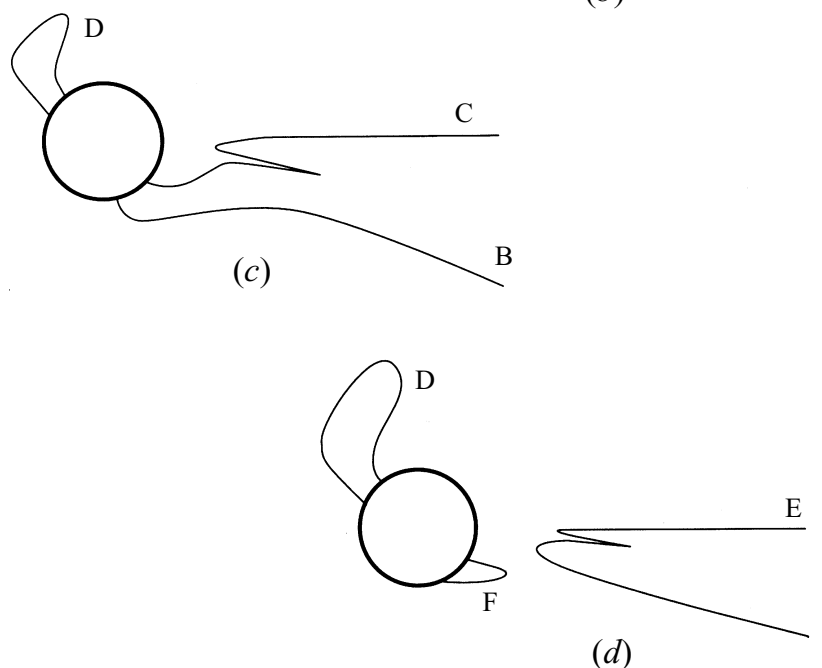

FIGURE 9. Schematic diagram of the topology of the zero vorticity lines at separation with $\beta$ increasing from $(a)$ to $(d)$.

\section{Results for the convected vortex}

Now consider the convected vortex problem with mainstream velocity distribution given by equation (3); the mainstream velocity is similar to that in the rotating cylinder problem in the sense that a region of favourable pressure gradient is followed by a zone of decelerated flow, but otherwise the two distributions are quite different. The main objective here is to determine whether separation is suppressed in the same manner as the cylinder problem; consequently, only the one numerical method described below was used, and no attempt was made to determine the precise critical value $\alpha_{c}$ (or alternatively $\beta_{c}$ ) at which unsteady separation is suppressed. An impulsive start was assumed and the initial development of the unsteady boundary-layer flow was calculated in Eulerian coordinates, until a switchover time $t_{o}=0.6$ to Lagrangian coordinates; a spatial mesh of $(201 \times 101)$ and a time step $\Delta t=10^{-3}$ were utilized. The flow development beyond $t=0.6$ was calculated in Lagrangian coordinates with a remeshing at $t=0.9$ for $\alpha=0.7$; the same number of mesh points was used with a time step of $\Delta t=5 \times 10^{-4}$, except for the calculations beyond the remesh at $t_{1}=0.9$ for which a finer mesh size of $(401 \times 201)$ was employed. In order to map the infinite streamwise domain onto a finite region, the following transformation was adopted in 

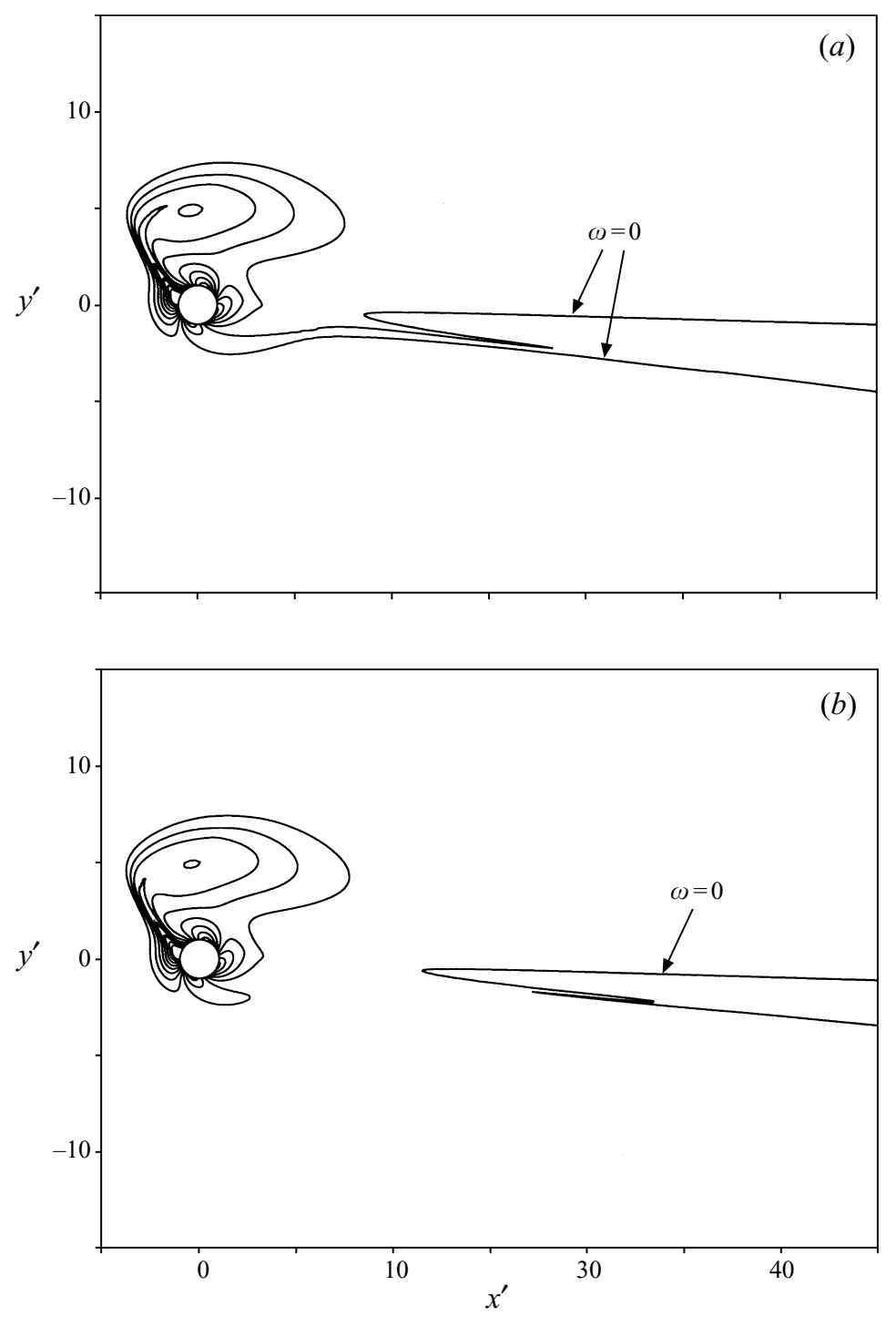

FIgURE 10. Calculated equivorticity lines at separation for $(a) \beta=1.8,(b) \beta=1.85$.

both Eulerian and Lagrangian coordinate formulations:

$$
x=h_{x}(\widehat{x})=\tan \left[\frac{1}{2} \pi(2 \widehat{x}-1)\right], \quad 0 \leqslant \widehat{x} \leqslant 1,
$$

with an analogous transformation for $\xi$.

The computed results for several values of $\alpha$ were found to agree with the Eulerian calculations of Doligalski \& Walker (1984), and the focus here is to extend the computations further in time using the Lagrangian formulation. The computed values of the location, instant and speed of unsteady separation are summarized in table 4 for increasing values of $\alpha$ (and hence the wall speed, $\beta$ ). With the mesh system adopted in this study, no unsteady separation was detected for values of $\alpha \geqslant 0.735$. As in the cylinder problem, the streamwise extent of the curve $\partial \widehat{x} / \partial \widehat{\xi}=0$ becomes increasingly narrower as $\alpha$ increases beyond 0.7 (cf. figure 4). Consequently, to determine the 


$\begin{array}{rllllllllll}\alpha & 0.5 & 0.55 & 0.6 & 0.65 & 0.67 & 0.7 & 0.71 & 0.72 & 0.73 & 0.735 \\ \beta & 1 & 1.22 & 1.5 & 1.86 & 2.03 & 2.33 & 2.45 & 2.57 & 2.70 & 2.77 \\ x_{s} & -0.484 & -0.557 & -0.661 & -0.824 & -0.922 & -1.13 & -1.21 & -1.29 & -1.35 & -1.39 \\ t_{s} & 0.783 & 0.817 & 0.875 & 0.985 & 1.08 & 1.22 & 1.24 & 1.27 & 1.30 & 1.35 \\ u_{s} & 0.27 & 0.29 & 0.16 & 0.058 & -0.011 & -0.19 & -0.23 & -0.34 & -0.36 & -0.35\end{array}$

TABLE 4. Calculated values of the location, instant and speed of unsteady separation singularity for the convected vortex flow.

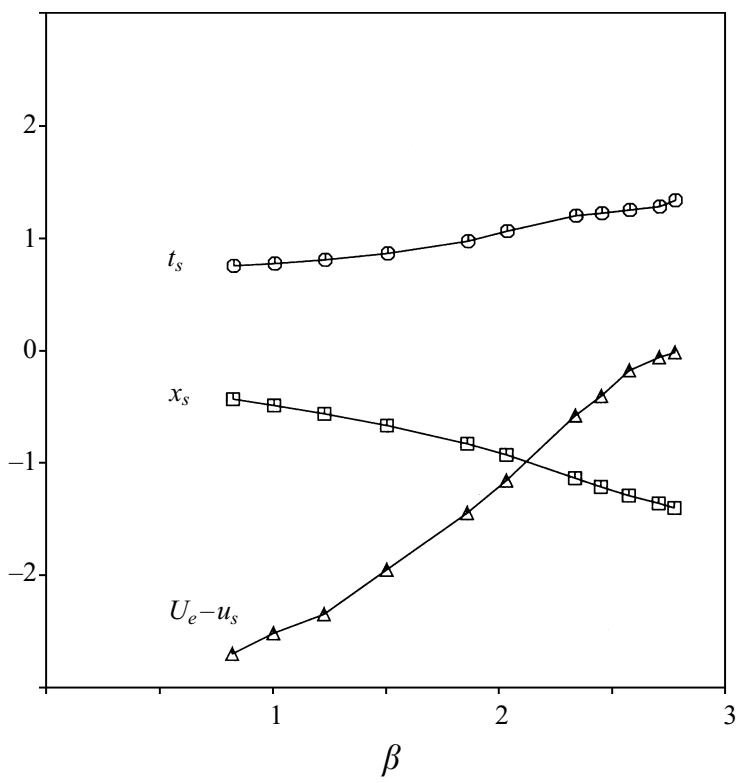

FIGURE 11. Variation of $x_{s}, t_{s}, U_{e}\left(x_{s}\right)-u_{s}$ with wall speed $\beta$ for the convected vortex problem.

critical value $\alpha_{c}$ more precisely, it would be necessary to adopt a transformation which sufficiently resolves the region where the singularity is expected (as described for the cylinder problem).

The results in table 4 are plotted in figure 11 where the close similarity with the results for the cylinder problem are apparent. The unsteady separation location $x_{s}$ moves toward the inviscid stagnation point at $x=-\sqrt{3}$ but does not reach this point before separation is suppressed; also the time of unsteady separation $t_{s}$ is delayed with increasing wall speed $\beta$. As in the cylinder problem, the magnitude of $U_{e}\left(x_{s}\right)-u_{s}$ decreases with increasing wall speed and approaches zero. Consequently, the calculations for the convected vortex problem also indicate that the limiting velocity profile at the point of suppression of unsteady separation is as shown in figure 6(a). Although a precise value of $\alpha_{c}$ was not determined, the calculations do indicate that unsteady separation is suppressed before $\alpha=0.75$ (corresponding to $\beta=3$ ). Consequently, as was the case for the cylinder problem, unsteady separation is suppressed when the wall speed is just less than the maximum value of the external flow velocity (in this case $\beta=3$ ).

Figure 12 shows the streamline patterns at $t_{s}-\Delta t$ for values of $\alpha=0.55,0.65$ and 0.7 . For all three values of $\alpha$, the streamlines may be observed to converge in a 

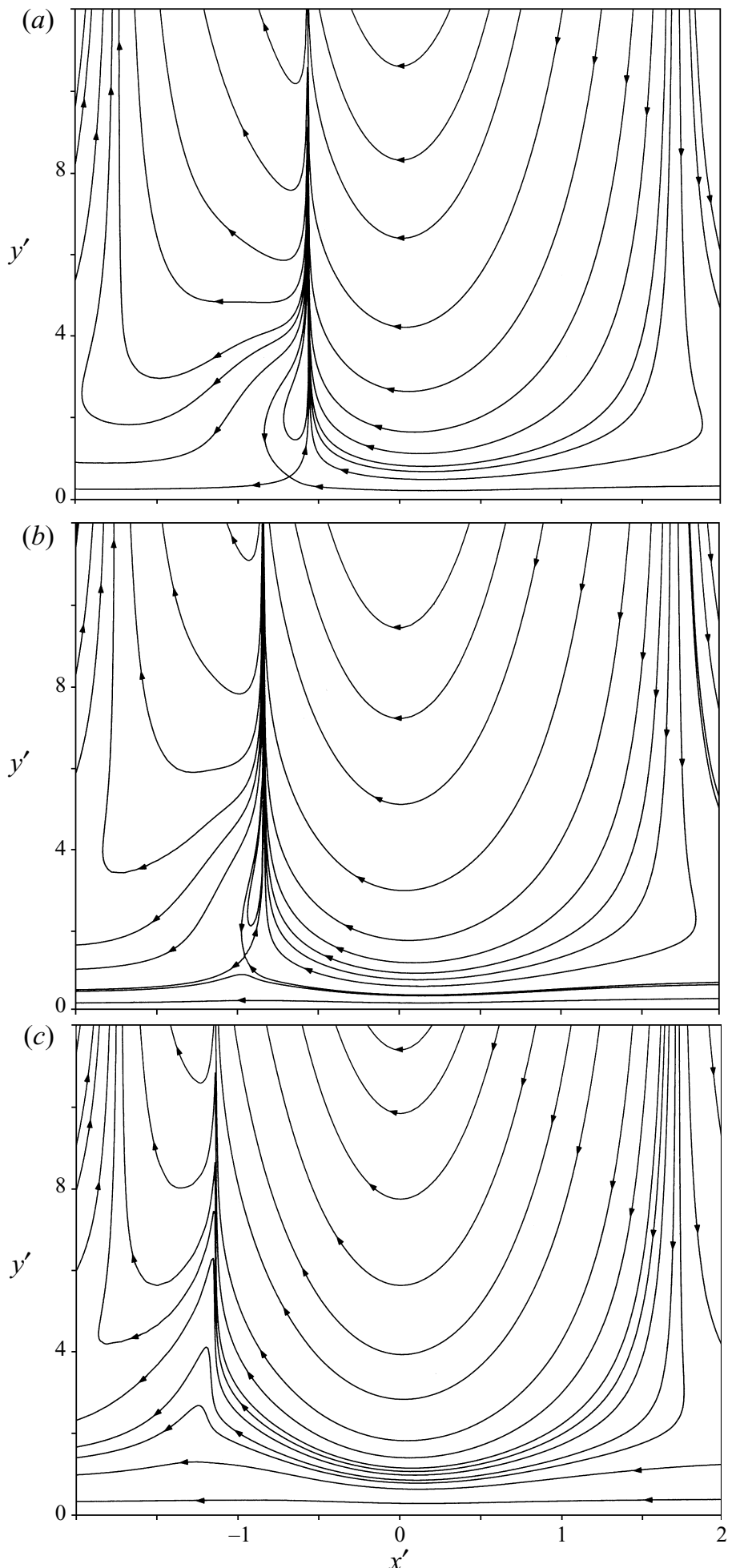

FIGURE 12. Instantaneous streamlines for the convected vortex problem at separation for $(a) \alpha=0.55,(b) \alpha=0.65,(c) \alpha=0.7$. 
narrow streamwise region at the location of the unsteady separation. For the lower value of $\alpha=0.55$, the separation point is located just upstream of the recirculating region (note that the mainstream flow is right to left between the two stagnation points located at $x= \pm \sqrt{3})$ as seen in figure 12(a). As the value of $\alpha$ increases, the streamwise extent of the recirculating region at the instant of separation decreases, as typified by the streamline pattern for $\alpha=0.65$ in figure $12(b)$ and as described in Appendix C. The formation of the recirculating region and the associated saddle point is suppressed for larger values of $\alpha$. Instead a weak 'jet-like' flow pattern develops as seen in figure $12(c)$ for $\alpha=0.7$. It may be observed that the change in the terminal streamline patterns with increasing wall speed is very similar for both the cylinder and convected vortex problems.

\section{Conclusions}

The unsteady boundary-layer flow past moving surfaces has been investigated here in the context of two model problems, namely the translating and rotating circular cylinder and a vortex convecting past an infinite flat plate. In the initial stages of the development of the flow, the traditional Eulerian formulation was used and at a predetermined time, the calculations were continued further in the Lagrangian formulation. Although the Lagrangian method provides an unambiguous criterion to determine the instant and location of the unsteady separation singularity, a difficulty associated with the formulation is mesh distortion caused by rapid separation of adjacent particles, which is most pronounced in regions of favourable pressure gradient. In the past, this difficulty was overcome by either concentrating mesh points in this region or by decreasing the time step substantially. In the present study, a remeshing algorithm was developed $(\S 4)$ whereby, after a predetermined number of time steps, an Eulerian description of the Lagrangian solution was obtained by solving the continuity equation and then using this to initiate the Lagrangian calculations once again. In this manner, the numerical difficulties due to mesh distortion were overcome, enhancing both computational speed and accuracy.

General trends for the development of unsteady separation past moving surfaces may be inferred from the calculated results for the two model problems considered here (in $\S 5$ and $\S 6$ ). The time of unsteady separation $t_{s}$ is delayed, and the location of unsteady separation $x_{s}$ approaches the inviscid stagnation point (but does not quite reach it) in the region of adverse pressure gradient as the surface speed $\beta$ increases. Both $t_{s}$ and $x_{s}$ appear to vary approximately linearly with surface speed $\beta$ as a critical value $\beta_{c}$ is approached, whereupon separation is suppressed. A referee has suggested that $t_{s}$ may tend to infinity as $\beta \rightarrow \beta_{c}$; the numerical results do not seem to support this, but this suggestion cannot be ruled out in view of the substantial numerical difficulties that occur in obtaining accurate solutions near $\beta_{c}$. The magnitude of $U_{e}\left(x_{s}\right)-u_{s}$, which may be considered to represent the strength of the separation phenomenon, decreases with increasing surface speed, and the calculations indicate that unsteady separation is suppressed at that critical value of surface speed where the magnitude of $U_{e}\left(x_{s}\right)-u_{s}$ becomes zero (see Appendix C). For the translating and rotating cylinder, $\beta_{c}$ is estimated to be 1.91 and for the convective vortex problem the estimate of $\beta_{c}$ is 2.77 (which corresponds to $\alpha_{c}=0.735$ ). An accurate determination of $\beta_{c}$ is rendered difficult because as $\beta$ approaches $\beta_{c}$, the streamwise extent of the curve $\partial x / \partial \xi=0$ becomes increasingly narrow. Consequently, this region of the flow must be increasingly well resolved in order to detect the unsteady separation singularity at all. It is interesting to note that in both of the model problems considered here, 
the critical value of $\beta_{c}$ is just less than the maximum magnitude of the external mainstream velocity associated with the region of adverse pressure gradient. In the cylinder problem, separation suppression occurs when the wall speed is $95.5 \%$ of the maximum inviscid flow speed (associated with the adverse pressure gradient), while the vortex problem indicates suppression at $92.3 \%$; the former value is a more accurate estimate, but whether a universal percentage is relevant in all unsteady flows is not known. However, at least for the model problems, it can be concluded that once the wall speed (i) is in the same direction as the maximum speed in the local inviscid flow (at a point where the region of adverse pressure gradient commences), and (ii) around $95 \%$ of the latter value, separation is effectively suppressed. At the critical value of the surface speed, the velocity of the separation structure just balances the local mainstream velocity. For all values of $\beta$ less than $\beta_{c}$, the separation velocity profile corresponds to the upstream-slipping. No evidence of downstream-slipping separation was found in either model problem.

For both model problems, a recirculation region develops in the flow at lower values of $\beta$. Subsequently, the unsteady separation singularity occurs just upstream of the recirculating region. On the other hand, at higher surface speeds, no recirculation is observed, but a weak jet-like outflow pattern develops prior to the occurrence of a considerably weakened separation singularity. A streamline pattern, suggested by Sears \& Telionis (1975) and Koromilas \& Telionis (1980) as characterizing 'upstreammoving wall' separation, was observed in the cylinder problem; however, the results in this study indicate that this flow pattern is not associated with unsteady separation.

It is of interest to comment on the physical mechanism through which separation and a strong interaction with the external flow is suppressed. Fluid particles in the boundary layer are subject to two important effects associated with the external pressure gradient and the moving wall. When the wall is stationary, fluid particles near the surface are slowed to rest to satisfy the no-slip condition at the surface; such particles are especially susceptible to the influence of the external pressure field, and in a zone of adverse pressure gradient, flow reversal, leading to zones of recirculation and ultimately boundary-layer separation, can easily occur. Once the wall moves, however, the fluid particles near the surface are energized and become less susceptible to the effects of the external pressure field. Separation can still occur under such circumstances, but it originates at an increasing distance from the wall with increasing wall speed where the particle speeds are generally smaller. Eventually, a growing wall speed dominates events in the boundary layer and strongly influences the separation speed $u_{s}$ in the central separating region (see Appendix C), forcing it to approach the local mainstream speed and thereby leading to the suppression of the separation effect.

The case $\beta=\beta_{c}$ corresponds to marginal separation as discussed by Cowley et al. (1990) who propose a general singularity structure for this type of separation. Cowley et al. (1990) showed that $t_{s}$ becomes infinite at a logarithmic rate in one case; thus it may be that here $\left|\beta-\beta_{c}\right|$ must be very small in order to see a significant increase in $t_{s}$. However, in view of the substantial difficulties in determining $\beta_{c}$ precisely, it was not possible to determine whether $t_{s} \rightarrow \infty$ as $\beta \rightarrow \beta_{c}$ in the present model problems; figures 5 and 11 suggest a slight increase in $t_{s}$ as $\beta \rightarrow \beta_{c}$ but are by no means definitive.

Lastly the question of what happens for $\beta>\beta_{c}$ is of interest. As previously remarked, steady periodic solutions for the rotating cylinder problem are available for $\beta \gtrsim \beta_{d}=3.91$ for an external flow with steady circulation. The present results show that the impulsively started problem terminates with a separation singularity 
in the range $0 \leqslant \beta<1.91$. Note that the two cylinder problems are similar but also different in that the issue of how the external flow acquires circulation for any finite $\beta_{c}$ is unresolved. A referee has suggested that calculations in the range $\beta_{c} \leqslant \beta<\beta_{d}$ should ultimately encounter another singularity at some larger but finite time. Our current computational experience does not support this. Calculations for all $\beta>\beta_{c}$ can be carried out for relatively long periods of time, but ultimately seem to encounter a persistent instability in the far wake at the end of the recirculation zone and near the downstream radius $x=\pi$. Grid refinement appears to hasten the onset of this instability and this is currently under investigation; however, the appearance of the instability precludes continued integration of the problem for $\beta>\beta_{c}$ on towards interaction with the external flow. Detailed results for this case will be reported elsewhere.

The authors would like to thank AFOSR for support of this work under Grant Number 91-0069. The authors would like to acknowledge a number of useful comments by the referees, and Stephen Cowley and Eric Varley.

\section{Appendix A. Functional coefficients}

In the Eulerian coordinate formulation, the coefficients in $(9 a)$ are given by

$$
\begin{gathered}
T=\frac{1}{\pi^{2} k_{\zeta}^{2} t} \cos ^{4}\left(\frac{1}{2} \pi \widehat{\zeta}\right), \\
Q=-\frac{u}{h_{x}^{\prime}} \\
P=\frac{1}{\pi^{2}} \cos ^{2}\left(\frac{1}{2} \pi \widehat{\zeta}\right)\left[\frac{2 \pi}{k_{\zeta} h_{x}^{\prime}} \frac{\partial \Psi}{\partial \widehat{x}}+\frac{\pi}{t} \tan \left(\frac{1}{2} \pi \widehat{\zeta}\right)\left\{1-\frac{\cos ^{2}\left(\frac{1}{2} \pi \widehat{\zeta}\right)}{k_{\zeta}^{2}}\right\}\right], \\
\Gamma=U_{e} \frac{\mathrm{d} U_{e}}{\mathrm{~d} x},
\end{gathered}
$$

where $h_{x}^{\prime}=\mathrm{d} h_{x} / \mathrm{d} \widehat{x}$.

In the Lagrangian coordinate formulation, the coefficients in (18) are given by

$$
\begin{aligned}
& R=\Pi\left(\frac{\partial \widehat{x}}{\partial \widehat{\eta}}\right)^{2} ; \quad S=-2 \Pi \frac{\partial \widehat{x} \widehat{\hat{\eta}}}{\frac{\partial}{\partial \widehat{\xi}}} ; \quad T=\Pi\left(\frac{\partial \widehat{x}}{\partial \widehat{\xi}^{2}}\right)^{2}, \\
& Q=\Pi\left[\frac{\partial \widehat{x}}{\partial \widehat{\eta}} \frac{\partial^{2} \widehat{x}}{\partial \widehat{\xi} \partial \hat{\eta}}-\frac{\partial^{2} \widehat{x}}{\partial \widehat{\eta}^{2}} \frac{\partial \widehat{x}}{\partial \widehat{\xi}}-\frac{h_{\xi}^{\prime \prime}}{h_{\xi}^{\prime \prime}}\left(\frac{\partial \widehat{x}}{\partial \widehat{\eta}}\right)^{2}+\pi \tan \left(\frac{1}{2} \pi \widehat{\eta}\right) \frac{\partial \widehat{x}}{\partial \widehat{\xi}} \frac{\partial \widehat{x}}{\partial \widehat{\eta}}\right], \\
& P=\Pi\left[\frac{\partial \widehat{x}}{\partial \widehat{\xi}} \frac{\partial^{2} \widehat{x}}{\partial \widehat{\xi} \partial \widehat{\eta}}-\frac{\partial^{2} \widehat{x}}{\partial \widehat{\xi}^{2}} \frac{\partial \widehat{x}}{\partial \widehat{\eta}}-\frac{h_{\xi}^{\prime \prime}}{h_{\xi}^{\prime}} \frac{\partial \widehat{x}}{\partial \widehat{\xi}} \frac{\partial \widehat{x}}{\partial \widehat{\eta}}-\pi \tan \left(\frac{1}{2} \pi \widehat{\eta}\right)\left(\frac{\partial \widehat{x}}{\partial \widehat{\xi}}\right)^{2}\right], \\
& \Gamma=U_{e} \frac{\mathrm{d} U_{e}}{\mathrm{~d} x}
\end{aligned}
$$

where

$$
\Pi=\frac{4}{\pi^{2}}\left(\frac{h_{x}^{\prime}}{k_{\eta} h_{\xi}^{\prime}}\right)^{2} \cos ^{4}\left(\frac{1}{2} \pi \widehat{\eta}\right),
$$


and

$$
h_{\xi}^{\prime}=\mathrm{d} h_{\xi} / \mathrm{d} \widehat{\xi}, \quad h_{\xi}^{\prime \prime}=\mathrm{d}^{2} h_{\xi} / \mathrm{d} \widehat{\xi}^{2}, \quad h_{x}^{\prime}=\mathrm{d} h_{x} / \mathrm{d} \widehat{x} .
$$

\section{Appendix B. Determination of singularity time}

In this Appendix, an algorithm to determine the instant, location and velocity of the unsteady separation singularity is described. At every time step, the solution is tested to determine if there is a location where $\partial \widehat{x} / \partial \widehat{\xi}$ and $\partial \widehat{x} / \partial \widehat{\eta}$ vanish simultaneously. This is accomplished by first evaluating $\partial \widehat{x} / \partial \widehat{\xi}$ and $\partial \widehat{x} / \partial \widehat{\eta}$ at each mesh point using second-order central difference formulae. Next, the entire mesh is swept to determine if both of the curves $\partial \widehat{x} / \partial \widehat{\xi}=0$ or $\partial \widehat{x} / \partial \widehat{\eta}=0$ simultaneously pass through any of the rectangular elements in the mesh as follows. Let $f=f(\widehat{\xi}, \widehat{\eta})$ denote either $\partial \widehat{x} / \partial \widehat{\xi}$ or $\partial \widehat{x} / \partial \widehat{\eta}$, and let $A=f_{i, j} f_{i+1, j+1}$ and $B=f_{i+1, j} f_{i, j+1}$ denote the products of functional values on diagonals for a typical cell in the mesh. If both $A$ and $B$ have the same sign, there are two possibilities: (i) the values of $f$ all have the same sign, in which case the curve $f=0$ either does not pass through the element or is entirely contained within it, and (ii) the signs of $f$ are different along each diagonal in which case the curve $f=0$ passes through the element at least twice. In the present algorithm, it is assumed that the mesh in the $(\widehat{\xi}, \widehat{\eta})$-plane is sufficiently small so that possibility (ii) may be discounted, not only because it is unlikely in a finely resolved mesh, but also because the error in estimating $t_{s}$ is small by allowing the solution to develop further in time so that one of the contours $f=0$ moves out of the mesh element in question.

If either $A$ or $B$ are negative, the contour $f=0$ passes through the mesh element once and in the algorithm is approximated within the element as a straight line. Let points within the mesh element having bottom left corner at $\left(\widehat{\xi}_{i}, \widehat{\eta}_{j}\right)$ be denoted by $\widehat{\xi}=\widehat{\xi}_{i}+p\left(\widehat{\xi}_{i+1}-\widehat{\xi}_{i}\right)$ and $\widehat{\eta}=\widehat{\eta}_{j}+q\left(\widehat{\eta}_{j+1}-\widehat{\eta}_{j}\right)$ where $0 \leqslant p, q \leqslant 1$; in effect $p, q$ define a coordinate system in the $(i, j)$ mesh cell. Then if either $A<0$ or $B<0$, two of the conditions in table 5 are satisfied and the intercepts of $f=0$ on the sides of the mesh element are defined by the values of $p$ and $q$ listed.

If the intercepts of $\partial \widehat{x} / \partial \widehat{\xi}=0$ are denoted by $\left(p_{\xi}^{1}, q_{\xi}^{1}\right)$ and $\left(p_{\xi}^{2}, q_{\xi}^{2}\right)$ and the intercepts of $\partial \widehat{x} / \partial \widehat{\eta}=0$ by $\left(p_{\eta}^{1}, q_{\eta}^{1}\right)$ and $\left(p_{\eta}^{2}, q_{\eta}^{2}\right)$, the equations for the approximating lines to $\partial \widehat{x} / \partial \widehat{\xi}=0$ and $\partial \widehat{x} / \partial \widehat{\eta}=0$ in the $(i, j)$ mesh cell are given by

$$
q=m_{\xi} p+C_{\xi}, \quad q=m_{\eta} p+C_{\eta},
$$

respectively, where

$$
m_{\xi}=\frac{q_{\xi}^{1}-q_{\xi}^{2}}{p_{\xi}^{1}-p_{\xi}^{2}}, \quad m_{n}=\frac{q_{\eta}^{1}-q_{\eta}^{2}}{p_{\eta}^{1}-p_{\eta}^{2}} ; \quad C_{\xi}=q_{\xi}^{1}-m_{\xi} p_{\xi}^{1}, \quad C_{\eta}=q_{\eta}^{1}-m_{\eta} p_{\eta}^{1} .
$$

These two lines intersect at a point defined by

$$
p_{s}=\frac{C_{\xi}-C_{\eta}}{m_{\eta}-m_{\xi}}, \quad q_{s}=m_{\eta} p_{s}+C_{\eta} .
$$

If $\left(p_{s}, q_{s}\right)$ are such that $0 \leqslant p_{s}, q_{s} \leqslant 1$, then a singularity is said to have been detected in the $(i, j)$ mesh cell at $\left(\widehat{\xi}_{s}, \widehat{\eta}_{s}\right)$ where

$$
\widehat{\xi}_{s}=\widehat{\xi}_{i}+p_{s}\left(\widehat{\xi}_{i+j}-\widehat{\xi}_{i}\right), \quad \widehat{\eta}_{s}=\widehat{\eta}_{j}+q_{s}\left(\widehat{\eta}_{j+i}-\widehat{\eta}_{j}\right),
$$

and the calculations are terminated. A third-order, six-point interpolation formula 


$\begin{array}{ccc}\text { Condition } & p & q \\ f_{i, j} f_{i+1, j} \leqslant 0 & a f_{i, j} & 0 \\ f_{i+1, j} f_{i+1, j+1} \leqslant 0 & 1 & a f_{i+1, j} \\ f_{i, j+1} f_{i+1, j+1} \leqslant 0 & a f_{i, j+1} & 1 \\ f_{i, j} f_{i, j+1} \leqslant 0 & 0 & a f_{i, j}\end{array}$

TABLE 5. Intercepts of $f=0$; for the product $f_{k, \ell} f_{m, n} a=\left(f_{k, \ell}-f_{m, n}\right)^{-1}$.

(Abramowitz \& Stegun 1972) was used to determine the value of $x$ and $u$ at $\left(\widehat{\xi}_{s}, \widehat{\eta}_{s}\right)$, hence providing the singularity location $x_{s}$ and velocity $u_{s}$.

In practice, this algorithm detects a singularity at two very close locations, one each in two adjacent elements. The reason for this is that the two curves $\partial \widehat{x} / \partial \widehat{\xi}=0$ and $\partial \widehat{x} / \partial \widehat{y}=0$ generally meet and then cross one another within one time step. In principal, the accuracy of the estimate may be increased by subdividing the final time step into progressively smaller time intervals; however, this is usually not necessary.

\section{Appendix C. Disappearance of the separation singularity}

In this Appendix, a brief theoretical account of the disappearance of the separation singularity as $\beta \rightarrow \beta_{c}$ is given. The structure of the boundary-layer solution near separation has been described by Van Dommelen (1981), Elliott et al. (1983) and Cassel et al. (1996) and may be summarized as follows. Assume a singularity occurs at $x_{s}$ and time $t_{s}$ and define

$$
\begin{aligned}
\widetilde{T}=t_{s}-t, \quad \widetilde{X} & =\frac{x-x_{s}-K\left(t_{s}-t\right)}{\left(t_{s}-t\right)^{3 / 2}}, \quad \widetilde{Y}=y\left(t_{s}-t\right)^{1 / 4}, \\
u & =-K+\left(t_{s}-t\right)^{1 / 2} \widetilde{U}(\widetilde{X}, \widetilde{Y}) .
\end{aligned}
$$

The above describe the streamwise velocity field in a central inviscid region bounded by a shear layer near the wall and one in the upper part of the boundary layer. As $t \rightarrow t_{s}$, the coordinate system moves upstream (for $K>0$ ) and a focusing occurs in physical space near $x_{s}$. The solution for the perturbation velocity $\widetilde{U}$ may be expressed in a number of ways and is of the form

$$
\frac{|\widetilde{U}|^{3 / 2}}{\widetilde{\tau}}= \pm G(\widetilde{\phi}), \quad \widetilde{\phi}=-\frac{\widetilde{U}^{3}}{\widetilde{U}+\widetilde{X}},
$$

where $\widetilde{\tau}=\partial \widetilde{U} / \partial \widetilde{Y}$ is the shear stress and

$$
G=\Lambda\left(\frac{|\widetilde{\phi}|}{\left|\widetilde{\phi}-\phi_{o}\right|}\right)^{1 / 2} .
$$

Here the constants $\Lambda$ and $\phi_{o}$, as well as $x_{s}$ and $t_{s}$, can only be evaluated, in general, from a global numerical solution. As described by Cassel et al. (1996), this solution leads to an implicit relation for $\widetilde{U}$ of the form

$$
\widetilde{Y}-\widetilde{Y}_{o}(\widetilde{X})= \pm \int_{\tilde{U}_{o}}^{\widetilde{U}} \frac{\mathrm{d} \xi}{\left\{\xi^{3}+\phi_{o}(\xi+\widetilde{X})\right\}^{1 / 2}},
$$




\begin{tabular}{ccccccccc}
\hline$\beta$ & $x_{s}$ & $U_{e}\left(x_{s}\right)$ & $u_{s}=-K$ & $u_{m}$ & $y_{b}$ & $y_{t}$ & $y_{m}$ & $y_{e}$ \\
1.6 & 3.41 & -0.53 & $\simeq 0$ & -0.24 & 9.4 & 41.6 & 51.5 & 56.8 \\
1.7 & 3.34 & -0.394 & -0.039 & -0.23 & 10.26 & 49.2 & 56.8 & 63.3 \\
1.8 & 3.27 & -0.256 & -0.12 & -0.23 & 9.63 & 56.8 & 67.1 & 76.2 \\
1.9 & 3.20 & -0.117 & -0.12 & -0.12 & 15.6 & large & large & large
\end{tabular}

TABLE 6. Raw data estimates of solution parameters near separation for problem described in $\S 5$.

where the + and - signs apply for $\widetilde{Y}>\widetilde{Y}_{o}$ and $\widetilde{Y}<\widetilde{Y}_{o}$, respectively; here $\widetilde{Y}_{o}(\widetilde{x})$ denotes a central line (where $\widetilde{\tau}=0$ ) that bisects the separating portion of the boundary layer and

$$
\widetilde{Y}_{o}(\widetilde{x})=\int_{\widetilde{U}_{o}}^{\infty} \frac{\mathrm{d} \xi}{\left\{\xi^{3}+\phi_{o}(\xi+\widetilde{X})\right\}^{1 / 2}} .
$$

The equation of the location of the upper shear layer bounding the separating fluid is $\widetilde{Y}=2 \widetilde{Y}_{o}(\widetilde{x})$.

The constants $\Lambda$ and $\phi_{o}$ can be scaled out by transformations.

$$
\widetilde{X}=\phi_{o}^{1 / 2} X, \quad \widetilde{Y}=\Lambda \phi_{o}^{-1 / 4} Y, \quad \widetilde{U}=\phi_{o}^{1 / 2} U, \quad \widetilde{\phi}=\phi_{o} \phi,
$$

and it is easily shown that equations (C 3)-(C 7) apply with the tildes removed and with $\phi_{o}=1$. It follows from equations (C2) and (C 7) that if $\phi_{o} \rightarrow 0$, the separation singularity is suppressed. The numerical results suggest that as $\beta \rightarrow \beta_{c}$, the separation singularity disappears; the results of $\S 5$ are the most detailed of the two problems considered, and from these, the critical value is estimated to be $\beta_{c} \simeq 1.91$. Velocity profiles at the instant prior to separation are shown in figure 4, and from the numerical results, the estimates shown in table 6 were obtained. Here $y_{b}$ denotes the location where the profile becomes flat nearest the wall; this value is a measure of the vertical extent of the lower shear layer bounding the central inviscid region. The quantity $u_{s}$ is the leading-order, almost uniform, velocity in the central region. In addition, $y_{t}, y_{m}$ and $y_{e}$ denote the approximate $y$ locations corresponding to: (i) the top of the central inviscid region (where the profile first deviates from the uniform central value of $-K$ ), (ii) the middle of the upper shear layer, and (iii) the top of the upper shear layer (where the profile again becomes uniform and almost equal to $U_{e}\left(x_{s}\right)$ ), respectively; $u_{m}$ denotes the velocity in the middle of the upper shear layer at $y=y_{m}$. It may be noted that some of these quantities are very difficult to estimate from the numerical solution with good accuracy, particularly as $\beta \rightarrow \beta_{c}$; for $\beta=1.9$, it can only be said with any certainty that $y_{t}, y_{m}$ and $y_{e}$ are very large.

The constant $\phi_{o}$ can be related to certain spatial and temporal derivatives of $x(\xi, \eta, t)$ from the Lagrangian solution at separation using the results detailed in Cowley et al. (1990), but it is very difficult to estimate $\phi_{o}$ accurately in this way. However the clear suggestion of the numerical results is that $\phi_{o} \rightarrow 0$ as $\Delta=\beta_{c}-\beta \rightarrow 0$. In view of equations (C 7), this explains why, as $\Delta \rightarrow 0$ : (i) the separation appears to weaken, (ii) the zone of separation is confined to a thinner region in the $x$-direction, thereby requiring the complicated remeshing algorithms, and (iii) the separation occurs further from the wall and over an expanded vertical direction. For the scalings shown in (C 7) to apply, then each of the ratios shown in table 7 should remain approximately constant for a fixed small value of $t_{s}-t$. It may be observed from table 7 that the 


$\begin{array}{ccccc}\Delta & \Delta^{1 / 4} y_{b} & \Delta^{1 / 4} y_{t} & \left(u_{s}-u_{m}\right) \Delta^{-1 / 2} & y_{m}^{2}\left(u_{s}-u_{m}\right) \\ 0.31 & 7.0 & 31 & 0.43 & 415 \\ 0.21 & 6.9 & 33 & 0.42 & 462 \\ 0.11 & 5.5 & 33 & 0.33 & 355 \\ 0.01 & 4.9 & - & - & -\end{array}$

TABLE 7. Comparison of the numerical results of $\S 4$ with the expected terminal singularity structure.

agreement with the proposed terminal structure is quite reasonable, even within the considerable limitations of this comparison.

The solution (C 3) and (C 4) is independent of the local pressure gradient as the term associated with this quantity becomes small as $\widetilde{T} \rightarrow 0$; thus the separating boundary layer 'forgets' the external pressure field which initially provoked the separation. To investigate the potential influence of the pressure gradient, assume that $\widetilde{U}$ in equation (C2) is also a function of $\widetilde{T}$. Using a similar development as in Cassel et al. (1996), it can be shown that in Crocco variables $\widetilde{\tau}=\widetilde{\tau}(\widetilde{X}, \widetilde{U}, \widetilde{T})=\partial \widetilde{U} / \partial \widetilde{Y}$ satisfies

$$
\left(\frac{3}{2} \widetilde{X}+\widetilde{U}\right) \frac{\partial \widetilde{\tau}}{\partial \widetilde{X}}+\left\{\frac{1}{2} \widetilde{U}-\mu(\widetilde{T})^{1 / 2}\right\} \frac{\partial \widetilde{\tau}}{\partial \widetilde{U}}-\widetilde{T} \frac{\partial \widetilde{\tau}}{\partial \widetilde{T}}=\frac{3}{4} \widetilde{\tau},
$$

where

$$
\mu=-U_{e} \frac{\partial U_{e}}{\partial x}=\frac{\partial p_{e}}{\partial x}
$$

is the pressure gradient evaluated at $x=x_{s}$. It may be deduced that the general solution of this equation is of the form

$$
\begin{gathered}
\frac{|\widetilde{U}-\mu \widetilde{T}|^{3 / 2}}{\widetilde{\tau}}=G(\widetilde{\alpha}, \widetilde{\phi}), \\
\widetilde{\alpha}=\widetilde{U} \widetilde{T}^{1 / 2}-\mu \widetilde{T}, \quad \widetilde{\phi}=-\frac{\left(\widetilde{U}-\frac{1}{2} \mu \widetilde{T}^{1 / 2}\right)^{3}}{\widetilde{X}+\widetilde{U}-\frac{1}{2} \mu \widetilde{T}^{1 / 2}} .
\end{gathered}
$$

For $\widetilde{T} \rightarrow 0, \widetilde{\alpha} \rightarrow 0$ and $G$ must approach equation (C4). Define

$$
U=\phi_{o}^{1 / 2}\left\{\widetilde{U}-\mu T^{1 / 2}\right\}, \quad T=\phi_{o}^{-1} \widetilde{T},
$$

along with the remaining scalings defined in equation (C 7). It follows that $\widetilde{\alpha}=O\left(\phi_{0}\right)$ as $\phi_{o} \rightarrow 0$ and that the unsteady solution is

$$
u=-K+\mu \phi_{o} T+\phi_{o}^{1 / 2} T^{1 / 2} U(X, Y, T)+\ldots,
$$

where $U$ satisfies

$$
|U|^{3 / 2} / \tau= \pm G(\phi), \quad \phi=-\frac{U^{3}}{U+X+\frac{1}{2} \mu T^{1 / 2}},
$$

$\tau=\partial U / \partial Y$ and $G$ is given by equation (C4) (with $\Lambda=\phi_{o}=1$ ).

Consider now a simple Taylor expansion of the free-stream condition $u \rightarrow U_{e}(x)$ for $\widetilde{T}$ fixed and small; using equation (C1) and $\widetilde{T}=\phi_{o} T$, this yields

$$
u \rightarrow U_{e}\left(x_{s}\right)-K \phi_{o} T U_{e}^{\prime}\left(x_{s}\right)+O\left(\phi_{o}^{3 / 2} T^{3 / 2}\right),
$$


which describes the solution above the upper shear layer in the separation structure. On the other hand, equation $(\mathrm{C} 13)$ describes the solution in the central region below the upper shear layer. It is evident that the two expressions agree as $\phi_{o} \rightarrow 0$ provided

$$
K=-U_{e}\left(x_{s}\right) .
$$

Consequently, the velocity in the central zone and the mainstream velocity must be in the same direction for this situation to occur, and the upper shear layer then vanishes as indicated in figures 3 and 4. The apparent unsteadiness in the first two terms in equation (C 13) is actually the (steady) free-stream behaviour in the local moving frame.

It is of interest to consider the solution in the shear layers bounding the central region where the motion is governed by the classical boundary-layer equations

$$
\frac{\partial u}{\partial t}+u \frac{\partial u}{\partial x}+\widetilde{v} \frac{\partial u}{\partial \widetilde{y}}=-\frac{\partial p_{e}}{\partial x}+\frac{\partial^{2} u}{\partial \widetilde{y}^{2}}, \quad \frac{\partial u}{\partial x}+\frac{\partial \widetilde{v}}{\partial \widetilde{y}}=0 .
$$

For the bottom shear layer $\widetilde{y}=y, \widetilde{v}=v$, while for the upper shear layer

$$
\widetilde{y}=y-\beta(x, t), \quad \widetilde{v}=v-u \frac{\partial \beta}{\partial x}-\frac{\partial \beta}{\partial t},
$$

with $\beta(x, t)=2 \widetilde{Y}_{o}(\widetilde{X})\left(t_{s}-\widetilde{t}\right)^{-1 / 4}$ denoting the equation of the moving upper shear layer. The solution in each shear layer is effectively of the form

$$
\begin{aligned}
u & =u_{o}(\widetilde{y})+\left(t_{s}-t\right)^{3 / 2} u_{1}(\widetilde{X}, \widetilde{y})+\ldots \\
\psi & =\psi_{o}(\widetilde{y})+\left(t_{s}-t\right)^{3 / 2} \psi_{1}(\widetilde{X}, \widetilde{y})+\ldots,
\end{aligned}
$$

where $\tilde{X}$ is defined by equations $(\mathrm{C} 1)$. Here $u_{i}=\partial \psi_{i} / \partial y$ and $u_{o}$ and $\psi_{o}$ are functions which are dependent upon the initial conditions (Cowley et al. 1990), but must satisfy $u_{o}=\psi_{o}$ at $\widetilde{y}=0$ and $u_{o} \sim-K+4 / \widetilde{y}^{2}$ as $\widetilde{y} \rightarrow \infty$ for the lower shear layer; for the upper shear layer

$$
u_{o} \sim-K+\frac{4}{\widetilde{y}^{2}} \quad \text { as } \quad \tilde{y} \rightarrow-\infty ; \quad u_{o} \sim U_{e}\left(x_{s}\right) \quad \text { as } \quad \tilde{y} \rightarrow \infty .
$$

Upon substitution in equation (C 17), it may be confirmed that

$$
\left(K+u_{o}\right) \frac{\partial^{2} \psi_{1}}{\partial \widetilde{y} \partial \widetilde{X}}-\frac{\partial \psi_{1}}{\partial \widetilde{X}} u_{o}^{\prime}=-\mu+u_{o}^{\prime \prime},
$$

which has the solution

$$
\frac{\partial \psi_{1}}{\partial \widetilde{X}}=\left(K+u_{o}\right) \int_{0}^{\tilde{y}} \frac{\left(u_{o}^{\prime \prime}-\mu\right)}{\left(u_{o}+K\right)^{2}} \mathrm{~d} \widetilde{y}+C\left(u_{o}+K\right) .
$$

For the lower shear layer, $C=0$ to satisfy the solid wall condition, and for the upper shear layer, $C$ is a function of $\widetilde{X}$, which may be related to displacement effects of the layers below. It may be verified that $\partial \psi_{1} / \partial \widetilde{X} \sim-\mu \widetilde{y}^{3} / 20+\ldots$ as $\widetilde{y} \rightarrow-\infty$, giving rise to a lower-order term $O\left(t_{s}-t\right)$ in the central region.

Now suppose that as $\beta \rightarrow \beta_{c}, u_{o}+K$ becomes almost uniform and small in the upper shear layer, namely $u_{o}+K=O(\widetilde{\varepsilon})$ for $\widetilde{\varepsilon}$ small, as suggested by the results in figure 4. It follows from (C 23) that $\partial \psi_{1} / \partial \widetilde{X} \sim-\mu \widetilde{\varepsilon}^{-1} \widetilde{X}$ in the upper shear layer. As $\beta \rightarrow \beta_{c}, \phi_{o} \rightarrow 0$ and with $t_{s}-t=O\left(\phi_{o}\right)$, it follows from (C7) that the expansion (C 19) becomes disordered when $\widetilde{\varepsilon}=O\left(\phi_{o}^{2}\right)$; in this circumstance, the upper shear layer becomes nonlinear as new physics come into play. 
It appears that a marginal separation structure exists as $\beta \rightarrow \beta_{c}$, the details of which may be similar to cases considered by Cowley et al. (1990). For $\beta>\beta_{c}$, an exact solution is given by $(\mathrm{C} 13)$ with $U=0$, and the solution is apparently free of singularities.

\section{REFERENCES}

Abramowitz, A. \& Stegun, I. 1972 Handbook of Mathematical Functions. Dover.

Bhaskaran, R., Smith, F. T. \& Timoshin, S. N. 1995 The appearance of boundary-layer instabilities as a velocity minimum develops. Proc. R. Soc. Lond. A 451, 331-339.

Cassel, K. W., Smith, F. T. \& Walker, J. D. A. 1996 The onset of instability in unsteady boundary-layer separation. J. Fluid Mech. 315, 233-256.

Chipman, P. D. \& Duck, P. W. 1993 On the high Reynolds number flow between non-coaxial rotating cylinders. Q. J. Mech. Appl. Maths 46, 163-192.

Cowley, S. J., Hocking, L. M. \& Tutty, O. R. 1985 The stability of solutions of the classical boundary-layer equations. Phys. Fluids 28, 441-443.

Cowley, S. J., Van Dommelen, L. L. \& Lam, S. T. 1990 On the Lagrangian variables in descriptions of unsteady boundary layer separation. Phil. Trans. R. Soc. Lond. A 333, 343-378.

Doligalski, T. L., Smith, C. R. \& Walker, J. D. A. 1994 Vortex interactions with walls. Ann Rev. Fluid Mech. 26, 573-616.

Doligalski, T. L. \& Walker, J. D. A. 1984 The boundary layer induced by a convected twodimensional vortex. J. Fluid Mech. 139, 1-28.

Ece, M. C., Walker, J. D. A. \& Doligalski, T. L. 1984 The boundary layer on an impulsively started rotating and translating cylinder. Phys. Fluids 27, 1077-1089.

Elliott, J. W., Smith, F. T. \& Cowley, S. J. 1983 Breakdown of boundary layers (i) on moving surfaces; (ii) in semi-similar unsteady flow; (iii) in fully unsteady flow. Geophys. Astrophys. Fluid Dyn. 25, 77-138.

Glauert, W. B. 1957 The flow past a rapidly rotating circular cylinder. Proc. R. Soc. Lond. A 242 , $108-115$

Koromilas, C. A. \& Telionis, D. P. 1980 Unsteady laminar separation: an experimental study. J. Fluid Mech. 97, 347-384.

LAM, S. T. 1988 On high-Reynolds-number laminar flows through a curved pipe, and past a rotating cylinder. PhD Thesis, Imperial College, University of London.

Moore, D. W. 1957 The flow past a rapidly rotating cylinder in a uniform stream. J. Fluid Mech. 2, 541-550.

Moore, F. K. 1958 On the separation of the unsteady laminar boundary layer. In Boundary Layer Research (ed. H. G. Gortler). Springer.

NikolayeV, K. V. 1982 Boundary-layer separation on a rotating cylinder in the flow of an incompressible fluid. Uch. Zap. TSAGI 13, 32-39.

Peridier, V. J., Smith, F. T. \& Walker, J. D. A. 1991 Vortex-induced boundary-layer separation. Part 1. The limit problem Re. J. Fluid Mech. 232, 99-131.

Rott, N. 1957 Unsteady viscous flow in the vicinity of a stagnation point. Q. J. Appl. Maths 13, 444-451.

Sears, W. R. 1956 Some recent developments in airfoil theory. J. Aeronaut. Sci. 23, 490-499.

Sears, W. R. \& Telionis, D. P. 1975 Boundary-layer separation in unsteady flow. SIAM J. Appl. Maths 28, 215-235.

Shen, S. F. \& WU, T. 1990 Unsteady separation over maneuvering bodies. AIAA J. 28, $2059-2068$.

Smith, C. R. \& Walker, J. D. A. 1995 Turbulent wall-layer vortices. In Fluid Vortices (ed. S. T. Green), pp. 235-290. Kluwer.

Smith, C. R., Walker, J. D. A., Haidari, A. H. \& Sobrun, U. 1991 On the dynamics of near-wall turbulence. Phil. Trans. R. Soc. Lond. A, 336, 131-175.

Smith, F. T. 1982 On the high Reynolds number theory of laminar flows. IMA J. Appl. Maths 28, $207-281$.

SycheV, V. V. 1987 Izv. Akad. Sci. USSR Mekh. Zhidk. Gaza, 43.

Telionis, D. P., Tsahalis, D. TH. \& Werle, M. J. 1973 Numerical investigation of unsteady boundary-layer separation. Phys. Fluids 16, 968-973. 
Telionis, D. P. \& Werle, M. J. 1973 Boundary-layer separation from downstream moving boundaries. Trans. ASME J. Appl. Mech. 95, 369-373.

Tsahalis, D. TH. 1977 Laminar boundary-layer separation from an upstream moving wall. AIAA J. 15, 561-566.

Van Dommelen, L. L. 1981 Unsteady boundary layer separation. PhD Thesis, Cornell University, Ithaca, NY.

Van Dommelen, L. L. 1991 Lagrangian Description of Unsteady Separation. Lecture Notes in Mathematics, vol. 28, pp. 701-718. Springer.

Van Dommelen, L. L. \& Cowley, S. J. 1990 On the Lagrangian description of unsteady boundary layer separation. J. Fluid Mech. 210, 593-626.

Van Dommelen, L. L. \& Shen, S. F. 1980 The spontaneous generation of the singularity in a separating laminar boundary layer. J. Comput. Phys. 38, 125-140.

Van Dommelen, L. L. \& Shen, S. F. 1982 The genesis of separation. In Proc. Symp. on Numerical and Physical Aspects of Aerodynamic Flows (ed. T. Cebeci). Springer.

VAn Dommelen, L. L. \& ShEn, S. F. 1983 Boundary-layer separation singularities for an upstream moving wall. Acta Mech. 49, 241-254.

Williams, J. C. 1977 Incompressible boundary-layer separation. Ann. Rev. Fluid Mech. 9, 113-144.

WU, T. 1991 Numerical studies of unsteady laminar boundary-layer separation over impulsivelystarted moving objects. PhD Thesis, Cornell University, Ithaca, NY. 